\title{
Manganese abundances in Galactic bulge red giants ${ }^{\star \star \star}$
}

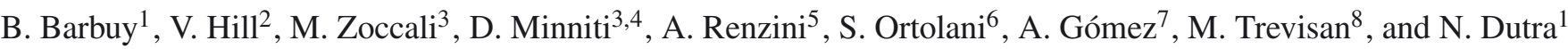 \\ 1 Universidade de São Paulo, IAG, Rua do Matão 1226, Cidade Universitária, 05508-900 São Paulo, Brazil \\ e-mail: barbuy@astro.iag.usp.br, nathalia.dutra@usp.br \\ 2 Université de Sophia-Antipolis, Observatoire de la Côte d'Azur, CNRS, Laboratoire Lagrange, BP4229, 06304 Nice Cedex 4, \\ France \\ e-mail: vanessa.hill@oca.eu \\ ${ }^{3}$ Instituto de Astrofisica, Facultad de Fisica, Pontificia Universidad Catolica de Chile, Casilla 306, 22 Santiago, Chile \\ e-mail: [mzoccali; dante]@astro.puc.cl \\ 4 Vatican Observatory, V00120 Vatican City State, Italy \\ 5 Osservatorio Astronomico di Padova, Vicolo dell’Osservatorio 5, 35122 Padova, Italy \\ e-mail: alvio.renzini@oapd.inaf.it \\ ${ }^{6}$ Università di Padova, Dipartimento di Astronomia, Vicolo dell'Osservatorio 2, 35122 Padova, Italy \\ e-mail: sergio.ortolani@unipd.it \\ 7 Observatoire de Paris-Meudon, 92195 Meudon Cedex, France \\ e-mail: anita.gomez@obspm.fr \\ 8 Instituto Nacional de Pesquisas Espaciais, Av. dos Astronautas 1758, 12227-010 São José dos Campos, Brazil \\ e-mail: marinatrevisan@gmail.com
}

Received 27 July 2013 / Accepted 18 September 2013

\section{ABSTRACT}

\begin{abstract}
Context. Manganese is mainly produced in type II SNe during explosive silicon burning, in incomplete Si-burning regions, and depends on several nucleosynthesis environment conditions, such as mass cut between the matter ejected and falling back onto the remnant, electron and neutron excesses, mixing fallback, and explosion energy. Manganese is also produced in type Ia SNe.

Aims. The aim of this work is the study of abundances of the iron-peak element Mn in 56 bulge giants, among which 13 are red clump stars. Four bulge fields along the minor axis are inspected. The study of abundances of Mn-over-Fe as a function of metallicity in the Galactic bulge may shed light on its production mechanisms.

Methods. High-resolution spectra were obtained using the FLAMES+UVES spectrograph on the Very Large Telescope. The spectra were obtained within a program to observe 800 stars using the GIRAFFE spectrograph, together with the present UVES spectra. Results. We aim at identifying the chemical evolution of manganese, as a function of metallicity, in the Galactic bulge. We find $[\mathrm{Mn} / \mathrm{Fe}] \sim-0.7$ at $[\mathrm{Fe} / \mathrm{H}] \sim-1.3$, increasing to a solar value at metallicities close to solar, and showing a spread around $-0.7 \lesssim[\mathrm{Fe} / \mathrm{H}] \lessgtr-0.2$, in good agreement with other work on $\mathrm{Mn}$ in bulge stars. There is also good agreement with chemical evolution models. We find no clear difference in the behaviour of the four bulge fields. Whereas $[\mathrm{Mn} / \mathrm{Fe}] \mathrm{vs}$. $[\mathrm{Fe} / \mathrm{H}]$ could be identified with the behaviour of the thick disc stars, $[\mathrm{Mn} / \mathrm{O}]$ vs. $[\mathrm{O} / \mathrm{H}]$ has a behaviour running parallel, at higher metallicities, compared to thick disc stars, indicating that the bulge enrichment might have proceeded differently from that of the thick disc.
\end{abstract}

Key words. stars: abundances - stars: late-type - Galaxy: abundances - Galaxy: bulge

\section{Introduction}

The Fe-peak elements include ( $\mathrm{Sc}), \mathrm{Ti}, \mathrm{V}, \mathrm{Cr}, \mathrm{Mn}, \mathrm{Fe}, \mathrm{Co}$, $\mathrm{Ni}, \mathrm{Cu}, \mathrm{Zn}, \mathrm{Ga}$, and $\mathrm{Ge}$, in a broad classification. The lighter iron group includes atomic mass elements in the range $22 \leq$ $Z \leq 26$, from titanium to iron (Woosley \& Weaver 1995, hereafter WW95). Despite being gathered in one category, iron-peak elements are produced in complex nucleosynthesis processes, therefore the abundances of some of these elements are not enriched in locksteps with $\mathrm{Fe}$. In particular, the elements $\mathrm{Sc}, \mathrm{Mn}$, $\mathrm{Cu}$, and $\mathrm{Zn}$ show different trends to that of Fe (e.g. Nissen et al. 2000; Ishigaki et al. 2013). We note that Sc has a behaviour intermediate between that of $\alpha$-elements and iron-peak elements, and its nucleosynthesis is not well established.

\footnotetext{
* Observations collected at the European Southern Observatory, Paranal, Chile (ESO programmes 71.B-0617A, 73.B0074A, and GTO 71.B-0196).

$\star \star$ Tables 1-6 and Figs. 1-6 are available in electronic form at http://www . aanda.org
}

The single manganese isotope ${ }^{55} \mathrm{Mn}$ is mainly produced in incomplete Si-burning regions, during explosive silicon burning in type II SNe (WW95) and in type Ia SNe (Bravo 2013). At peak temperature, $4 \times 10^{9}<T_{\text {peak }}<5 \times 10^{9} \mathrm{~K}$, the shocked material subsequently undergoes explosive nucleosynthesis in Si burning regions, and unstable ${ }^{55} \mathrm{Co}$ is produced, which decays into ${ }^{55} \mathrm{Mn}$. In core collapse $\mathrm{SNe}$ the amount of $\mathrm{Mn}$ ejected depends on the mass cut between the ejecta and the inner core (Nakamura et al. 1999). It also depends on the electron excess $Y_{\mathrm{e}}$ (and in turn the progenitor metallicity), neutron excess, mixing-fallback process, and explosion energy (Umeda \& Nomoto 2002, hereafter UN02; Kobayashi et al. 2006; Thielemann et al. 1996; WW95). Umeda \& Nomoto (2002) considered a $Y_{\mathrm{e}}$ larger by only 0.00001 in the incomplete Si-burning region of population III very low metallicity $\mathrm{SNe}$, and as a result noted that their $\mathrm{Mn}$ yields are smaller by a factor of 10 than the yields of Nakamura et al. (1999). These dependencies were also investigated by Umeda $\&$ Nomoto (2005) at very low metallicities. The production of 
Mn in incomplete Si-burning regions in SNe Ia is described in, e.g. Iwamoto et al. (1999) and Bravo (2013).

In the present work, we derive Mn abundances for a sample of 56 bulge field stars, observed at high spectral resolution with the FLAMES-UVES spectrograph at the Very Large Telescope. The sample consists of red giants in four bulge fields: Baade's Window $\left(l=1.14^{\circ}, b=-4.2^{\circ}\right)$, a field at $b=-6^{\circ}\left(l=0.2^{\circ}\right.$, $\left.b=-6^{\circ}\right)$, the Blanco field $\left(l=0^{\circ}, b=-12^{\circ}\right)$, and a field near NGC $6553\left(l=5.2^{\circ}, b=-3^{\circ}\right)$, as described in Lecureur et al. (2007) and Zoccali et al. (2006, 2008). The red clump stars in Baade's Window were analysed by Hill et al. (2011).

We compare our results to previous samples in the literature. McWilliam et al. (2003a,b) and Sobeck et al. (2006) derived Mn abundances in seven field and three NGC 6528 bulge giants, respectively. In order to try to understand the overall Mn chemical enrichment, the present results are also compared with the trends for thin disc, thick disc, and halo stars, derived by Gratton (1989), Prochaska et al. (2000), Reddy et al. (2003, 2006), Sobeck et al. (2006), Feltzing et al. (2007), Nissen \& Schuster (2011), and Cayrel et al. (2004).

We also compare the results with chemical evolution models that take into account the early enrichment by core collapse $\mathrm{SNe}$ and the later type Ia SNe that cause the increase in $[\mathrm{Mn} / \mathrm{Fe}]^{1}$ towards solar values (Cescutti et al. 2008; Timmes et al. 1995; Tsujimoto \& Shigeyama 1998).

In Sect. 2 the observations are reported. In Sect. 3 the atomic constants for the lines under study are given. In Sect. 4 the basic stellar parameters are listed, and the abundance derivation of $\mathrm{Mn}$ is described. The results are derived in Sect. 5 and discussed in Sect. 6. Conclusions are drawn in Sect. 7.

\section{Observations}

The spectra were obtained with the FLAMES-UVES spectrograph, at the $8.2 \mathrm{~m}$ Kueyen ESO telescope. The mean wavelength coverage is $4800-6800 \AA$, with a gap at $5775-5825 \AA$. The red portion of the spectrum (5800-6800 $\AA$ ) was obtained with the ESO CCD \# 20, an MIT backside illuminated, of $4096 \times 2048$ pixels, and pixel size $15 \times 15 \mu \mathrm{m}$. The blue portion of the spectrum (4800-5800 ̊) uses ESO Marlene EEV CCD\#44, backside illuminated, of $4102 \times 2048$ pixels, and pixel size $15 \times 15 \mu \mathrm{m}$. With the UVES standard setup 580, the resolution is $R \sim 45000$ for a 1 arcsec slit width, and $R \sim 55000$ for a slit of 0.8 arcsec. The pixel scale is $0.0147 \AA /$ pix, and typical signal-to-noise ratios obtained are in the range 9 to 70 per pixel, or 25 to 190 per resolution element ( 7.5 pixels at $6000 \AA)$.

The spectra were reduced using the FLAMES-UVES pipeline ${ }^{2}$, including bias and inter-order background subtraction, flatfield correction, extraction, and wavelength calibration (Modigliani et al. 2004).

The selection of bulge stars was carried out by choosing red giants at about one magnitude above the horizontal branch, as described in Zoccali et al. (2006). Red clump stars were selected by Hill et al. (2011). The list of stars is reported in Sect. 4.

\footnotetext{
1 We adopted here the usual spectroscopic notation that $[\mathrm{A} / \mathrm{B}]=$ $\log \left(\mathrm{N}_{\mathrm{A}} / \mathrm{N}_{\mathrm{B}}\right)_{\star}-\log \left(\mathrm{N}_{\mathrm{A}} / \mathrm{N}_{\mathrm{B}}\right)_{\odot}$ and $\epsilon(\mathrm{A})=\log \left(\mathrm{N}_{\mathrm{A}} / \mathrm{N}_{\mathrm{B}}\right)+12$ for each elements $\mathrm{A}$ and $\mathrm{B}$.

2 http://www . eso.org/sci/software/pipelines
}

\section{Line parameters: hyperfine structure, oscillator strengths, and solar abundances}

Hyperfine structure (hfs) in some lines, particularly in odd- $Z$ elements, are produced from interactions between nuclear and electronic wave functions. The imbalance of neutrons and protons produces a large nuclear magnetic moment.

The hfs desaturates strong absorption lines, resulting in broader lines and higher equivalent widths than if considered as single lines (e.g. Prochaska \& McWilliam 2000). Therefore neglecting hfs effects leads to overestimating abundances.

Manganese abundances were derived from the Mn I triplet lines at $6000 \AA$. We also measured the Mn I 5394.67 $\AA$ line for a few stars, and since this line is strong in giants, it was used particularly for the metal-poor and/or Mn-poor stars.

Central wavelengths were adopted from the National Institute of Standards and Technology (NIST, Martin et al. 2002), which appeared to be the most suitable ones for the location of lines in the solar and Arcturus spectra.

Table 2 shows the line excitation potential, $\log g f$ values from the literature, and adopted total $\log g f$-values for the four studied lines. Literature $g f$-values are from Booth et al. (1984), Blackwell-Whitehead \& Bergemann (2007, hereafter B-HB07), Den Hartog et al. (2011), and from the line lists by Kurúcz $(1993)^{3}$, NIST $^{4}$, and VALD (Piskunov et al. 1995). The adopted $g f$-values were obtained by fitting the solar high-resolution observations using the same UVES spectrograph as the present sample of spectra ${ }^{5}$, and Arcturus (Hinkle et al. 2000) spectra. Our astrophysical gf values are closer to those of B-HB07.

The hyperfine structure for the studied lines of Mn I was taken into account by employing a code made available by Andrew McWilliam, following the calculations described by Prochaska \& McWilliam (2000). The nuclear spin $(I=2.5)$ of the only nuclide that contributes for the manganese abundance $\left({ }^{55} \mathrm{Mn}\right)$ was found in Woodgate \& Martin (1957). For the MnI 6013/6016/6021 and 5394 lines, experimental data on hyperfine coupling constants, namely the magnetic dipole A-factor, and the electric quadrupole B-factor, were adopted from Handrich et al. (1969), Biehl (1976), and Brodzinski et al. (1987) (transformed from MKaysers $\left(\mathrm{cm}^{-1}\right)$ to $\mathrm{MHz}$ ), and are given in Table 1 . The hfs components for the Mn I lines and corresponding oscillator strengths are reported in Table 3. The present fits to the solar and Arcturus spectra for the Mn I lines are shown in Fig. 1.

The adopted solar abundances for $\mathrm{Fe}$ and $\mathrm{Mn}$ are from Grevesse \& Sauval (1998): $\left(\log \epsilon(\mathrm{Fe})_{\odot}, \log \epsilon(\mathrm{Mn})_{\odot}\right)=(7.50$, 5.39), in good agreement with those from Asplund et al. (2009) and Lodders et al. (2009), of (7.50, 5.43) and (7.46, 5.50) respectively.

For Arcturus we adopted the parameters from Meléndez et al. (2003) of $\left(T_{\mathrm{eff}}(\mathrm{K}), \log g,[\mathrm{Fe} / \mathrm{H}], v_{\mathrm{t}}\left(\mathrm{km} \mathrm{s}^{-1}\right)=(4275,1.55\right.$, $-0.54,1.65)$, which are in good agreement with those from Ramírez \& Allende Prieto (2011) of (4286, 1.66, -0.52, 1.74). For Arcturus, a $\mathrm{Mn}$ abundance of $[\mathrm{Mn} / \mathrm{Fe}]=-0.25$ fitted the Mn lines with the same parameters as for the Sun; this is in excellent agreement with the Mn abundance given by Ramírez \& Allende Prieto (2011) of $[\mathrm{Mn} / \mathrm{Fe}]=-0.21$.

\footnotetext{
http://kurucz.harvard. edu/atoms.html

4 http://physics.nist.gov/PhysRefData/ASD/lines_form. html

5 http://www . eso.org/observing/dfo/quality/UVES/

pipeline/solar_spectrum.html
} 


\section{Abundance analysis}

The list of stars is reported in Table 4, together with their stellar parameters.

\subsection{Atmospheric parameters and abundance calculation}

Manganese abundances were obtained through line-by-line spectrum synthesis calculations. The calculations of synthetic spectra were carried out using the code PFANT described in Barbuy et al. (2003). Molecular lines of the $\mathrm{CN} \mathrm{A}^{2} \Pi-\mathrm{X}^{2} \Sigma$, $\mathrm{C}_{2}$ Swan $\mathrm{A}^{3} \Pi-\mathrm{X}^{3} \Pi$, and TiO $\mathrm{A}^{3} \Phi-\mathrm{X}^{3} \Delta \gamma$ and $\mathrm{B}^{3} \Pi-\mathrm{X}^{3} \Delta \gamma^{\prime}$ systems are taken into account.

The MARCS local thermodynamic equilibrium (LTE) 1D model atmospheres grid was employed (Gustafsson et al. 2008). For the red giant stars, we used the parameters derived by Zoccali et al. (2006) and Lecureur et al. (2007), while for red clump stars we used the parameters updated by Hill et al. (2011). For these stars, Hill et al. (2011) carried out the analyses based both on GIRAFFE spectra and UVES spectra of the same stars, and we adopted the UVES stellar parameters.

Gaussian convolutions with $\sigma=2,3$, and 4, and even 5 pixels for the noisier spectra, with $0.0147 \AA$ /pixel, were applied to all spectra, to improve the fits to the faint lines studied in this work.

For the few metal-poor stars, we added the fit to the MnI $5394 \AA$ line, in order to be able to derive their Mn abundances more accurately. The fits to the four MnI lines for metalpoor sample stars are shown in Figs. 2-5. Mn abundances are reported in Table 4 , where $\mathrm{Mn} 1 / \mathrm{Mn} 2 / \mathrm{Mn} 3 / \mathrm{Mn} 4$ refer to the 6013/6016/6021/5934 A lines.

\subsection{Uncertainties}

The main uncertainties are those coming from the derivation of stellar parameters, as described in Lecureur et al. (2007), where the following uncertainties were adopted: $\pm 200 \mathrm{~K}$ for temperature, \pm 0.20 for surface gravity (photometric gravities derived assuming that the stars are at the distance of the bulge), \pm 0.1 for metallicity, and $\pm 0.10 \mathrm{~km} \mathrm{~s}^{-1}$ for microturbulence velocity. The profile fitting errors are to be added to these, estimated to be of the order of \pm 0.1 in Mn-over-Fe abundances. For eight sample stars, Ryde et al. (2010) carried out a re-analysis using the same equivalent widths measured by Lecureur et al. (2007). They found cooler temperatures by about $160 \mathrm{~K}$, and estimated errors in spectroscopic gravity of \pm 0.5 . Hill et al. (2011) reported errors in $[\mathrm{Fe} / \mathrm{H}]$ star-by-star for the GIRAFFE spectra. Based on the uncertainties on stellar parameters as discussed in Lecureur et al. (2007) and Ryde et al. (2010), we assumed uncertainties of $\pm 150 \mathrm{~K}$ in temperature, \pm 0.2 in $\log g$ given that we used photometric gravities, \pm 0.1 in $[\mathrm{Fe} / \mathrm{H}]$, and \pm 0.1 in microturbulence velocity. Table 5 reports the errors in $[\mathrm{Mn} / \mathrm{Fe}]$ due to a change in the parameters for two giants close to the edges of our $[\mathrm{Fe} / \mathrm{H}]$ range: the metal-poor star B3-B1 and the red clump star BWc-6. In this table we also report the different results by adopting the stellar parameters from the several analyses carried out as described above. The errors in $[\mathrm{Mn} / \mathrm{Fe}]$ are computed using model atmospheres corresponding to each of these models, and rederiving $[\mathrm{Mn} / \mathrm{Fe}]$ from fitting the observed spectra. The errors are the differences relative to the fitting with the adopted model. The overall uncertainties due to stellar parameters are given in Table 6 for these two sample stars. We note that these final errors are upper limits, given that the stellar parameters are covariant.
Table 7. Mean and dispersion of $[\mathrm{Mn} / \mathrm{Fe}]$ in bulge stars in four bins of metallicity.

\begin{tabular}{lrrr}
\hline \hline Bin & $\langle[\mathrm{Mn} / \mathrm{Fe}]\rangle$ & $\sigma_{\mathrm{Mn} / \mathrm{Fe}}$ & $\mathrm{N}_{\text {stars }}$ \\
\hline$-0.8 \leq[\mathrm{Fe} / \mathrm{H}]<-0.5$ & -0.13 & 0.15 & 4 \\
$-0.5 \leq[\mathrm{Fe} / \mathrm{H}]<-0.2$ & -0.09 & 0.12 & 11 \\
$-0.2 \leq[\mathrm{Fe} / \mathrm{H}]<+0.1$ & 0.00 & 0.03 & 16 \\
$+0.1 \leq[\mathrm{Fe} / \mathrm{H}]<+0.4$ & 0.00 & 0.07 & 22 \\
\hline
\end{tabular}

Notes. The last column gives the number of stars in each bin.

Finally, adding \pm 0.1 due to a continuum location uncertainty, we estimate a mean uncertainty in $[\mathrm{Mn} / \mathrm{Fe}]$ values of \pm 0.2 .

Non-LTE effects on Mn lines were computed by Bergemann \& Gehren (2008). While the effects are considerable in metalpoor stars hotter than $5000 \mathrm{~K}$, increasing with increasing temperature and decreasing metallicity, they appear to be below (nonLTE-LTE) $\lesssim+0.1$ dex for the metallicities and temperatures of the present sample stars. We preferred not to try to correct the literature Mn abundances for halo stars, given that non-LTE effects are counterbalanced by 3D modelling, therefore a correction taking into account both effects is not known.

\section{Results}

Table 4 reports the Mn abundances derived in the present work. In Fig. 7a our results are plotted with different symbols identifying each of the four fields and the red clump stars. The first conclusion that can be drawn is that there is no clear distinction in $\mathrm{Mn}$ abundances among the four bulge fields. Figure 7 clearly indicates that, from values of $[\mathrm{Mn} / \mathrm{Fe}] \sim-0.7$ at $[\mathrm{Fe} / \mathrm{H}] \sim-1.3$, $[\mathrm{Mn} / \mathrm{Fe}]$ increases to the solar value at the solar metallicity, although in the metallicity range $-0.7 \lesssim[\mathrm{Fe} / \mathrm{H}] \lesssim-0.2$ the trend is somewhat ill-defined because of the scarcity of data points and their relatively large dispersion. To understand whether this dispersion is true, in Fig. 8 we show our results for the bulge stars together with a moving average line (moving average on 10 points) and the corresponding rms dispersion in each subsample (shown as dashed lines). This plot indicates a larger dispersion of $[\mathrm{Mn} / \mathrm{Fe}]$ for metallicities below solar, and even larger for $[\mathrm{Fe} / \mathrm{H}]<-0.5$, although in this last part the scarcity of data may be the reason for the apparent increased dispersion (a large $[\mathrm{Fe} / \mathrm{H}]$ range enters the first few moving average samples, so that the slope in $\mathrm{Mn} / \mathrm{Fe}$ acts to increase the dispersion among the sample). Table 7 shows the mean $[\mathrm{Mn} / \mathrm{Fe}]$ values in four metallicity bins, together with their dispersion, and confirms that $[\mathrm{Mn} / \mathrm{Fe}]$ may be more dispersed at subsolar metallicities.

\subsection{Comparison with chemical evolution models}

Chemical evolution model predictions by Timmes et al. (1995) and Tsujimoto \& Shigeyama (1998, hereafter TS98) for the solar neighborhood, as well as Cescutti et al. (2008) for the Galactic bulge are also displayed in Fig. 7.

Timmes et al. (1995) carried out computations of chemical evolution models for the solar neighborhood, based on metallicity dependent yields by WW95. The nucleosynthesis results by WW95 consisted of 60 type II supernova models of masses $11 \lesssim M / M_{\odot} \lesssim 40$ and metallicities from zero to solar, incorporating 76 stable isotopes from $\mathrm{H}$ to $\mathrm{Zn}$. Timmes et al. also included yields from type Ia SNe, Big-Bang nucleosynthesis of the light elements, and mass loss from planetary nebulae. For $\mathrm{SNe}$ Ia, Timmes et al. followed the prescriptions by Matteucci \& Greggio (1986), adopting the results from SNe Ia models 

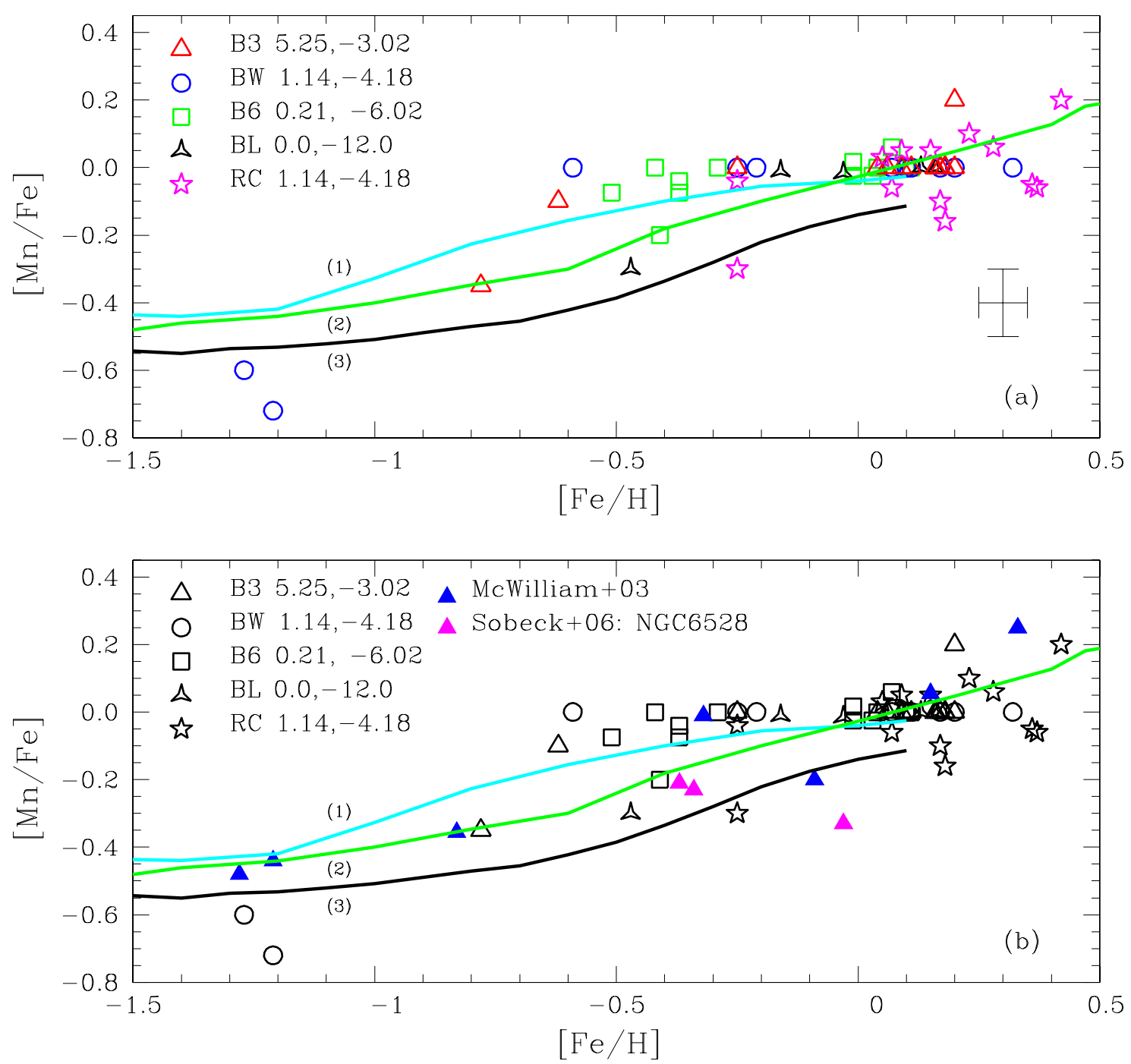

Fig. 7. $[\mathrm{Mn} / \mathrm{Fe}]$ vs. $[\mathrm{Fe} / \mathrm{H}]$ : a) present results for the four bulge fields. Symbols: open red triangles: B3 stars from the NGC 6553 field $\left(l=5.2^{\circ}\right.$, $\left.b=-3^{\circ}\right)$; open blue circles: BW stars from Baade's Window $\left(l=1.14^{\circ}, b=-4.2^{\circ}\right)$; open green squares: B6 stars from a field at $b=-6^{\circ}\left(l=0.2^{\circ}\right.$, $\left.b=-6^{\circ}\right)$; open black triangles: Bl stars from the Blanco field $\left(l=0^{\circ}, b=-12^{\circ}\right)$; open magenta stars: red clump (RC) stars from Baade's Window. An error bar is given in the bottom-right corner of Fig. 2a. b) Same as a), here including bulge field stars (full blue triangles) from McWilliam et al. (2003a,b), and NGC 6528 from Sobeck et al. (2006) (full magenta triangles). Solid lines correspond to chemical evolution models by (1) TS98 (blue); (2) Cescutti et al. (2008) (green); and (3) Timmes et al. (1995) (black).

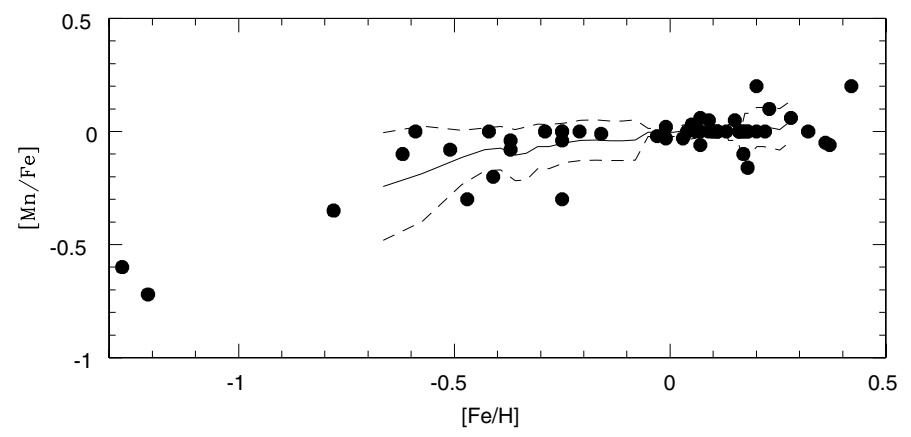

Fig. 8. Present results on $[\mathrm{Mn} / \mathrm{Fe}]$ vs. $[\mathrm{Fe} / \mathrm{H}]$ together with a moving average line (moving average on 10 points) and the corresponding rms dispersion in each subsample (shown as dashed lines).

by Nomoto et al. (1984) and the yields from Thielemann et al. (1986). No explicit dependence on metallicity was employed for the SNe Ia input data.
Tsujimoto \& Shigeyama (1998) employed the SNe II yields from Nomoto et al. (1997) and the chemical evolution model by Yoshii et al. (1998). There is no particular consideration of yields as a function of metallicity. The TS98 models imply low $[\mathrm{Mn} / \mathrm{Fe}]$ values for metallicities $[\mathrm{Fe} / \mathrm{H}] \lesssim-2.8$, and a Mn plateau at $-2.8 \lesssim[\mathrm{Fe} / \mathrm{H}] \lesssim-1.0$, because the most massive stars eject about 2 orders of magnitude larger mass of Mn than do the lower mass $\mathrm{SNe}$ II. For $[\mathrm{Fe} / \mathrm{H}]>-1$ the $\mathrm{SN}$ Ia cause a clear increase in the $[\mathrm{Mn} / \mathrm{Fe}]$ values. Both Timmes et al. and TS98 models assume a Salpeter initial mass function (IMF).

Cescutti et al. (2008) computed models specifically for the Galactic bulge, by assuming a flatter IMF and a star formation efficiency 20 times higher than the solar vicinity, as previously established by Ballero et al. (2007). Their most suitable model option involves metallicity dependent yields from WW95 for type II SNe, combined with metallicity dependent yields from type Ia SNe, that were obtained by applying a dependence law proportional to the overall metallicity $Z$, of $Z^{0.65}$, to yields from Iwamoto et al. (1999). 
B. Barbuy et al.: Manganese abundances in Galactic bulge red giants
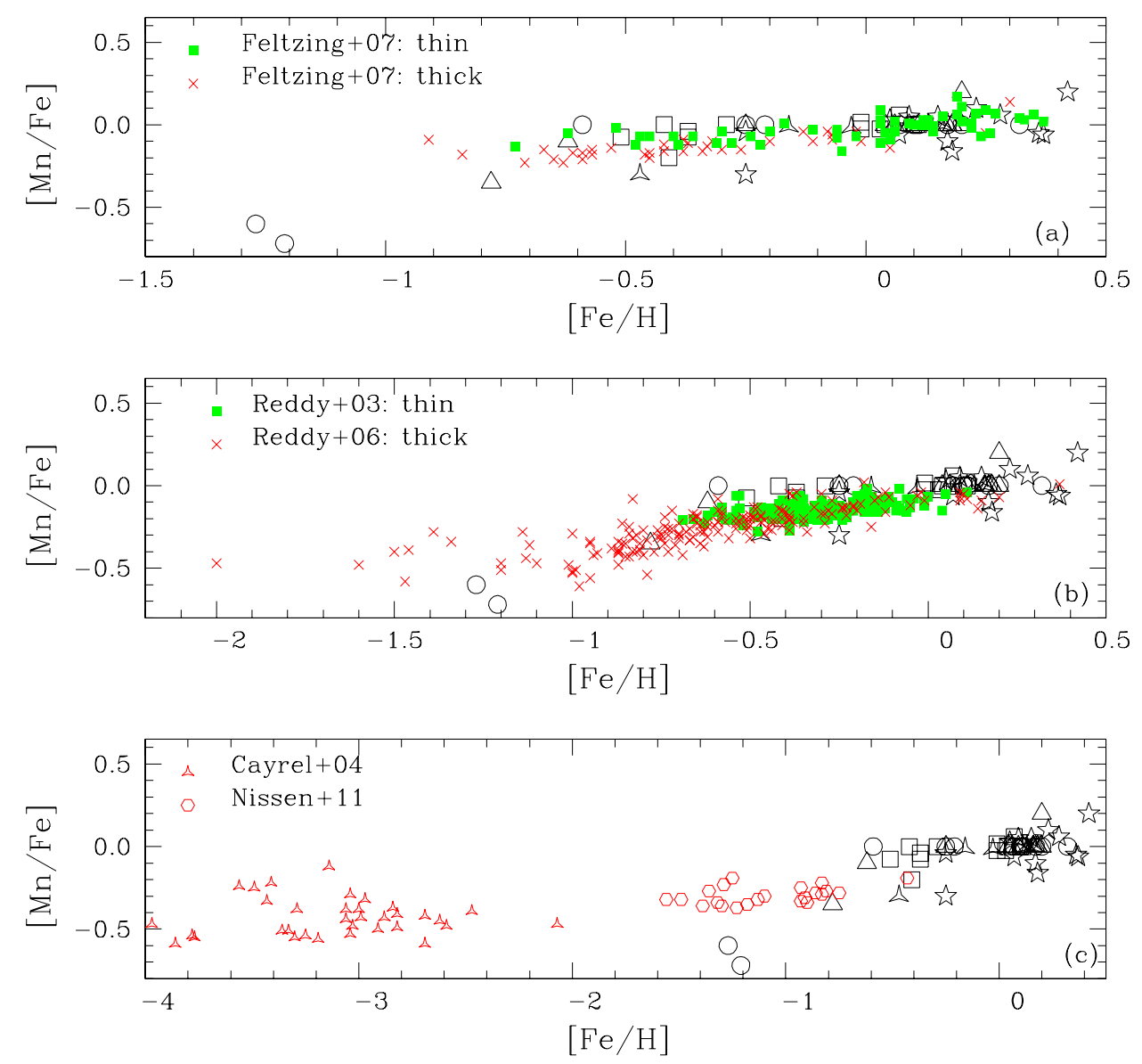

Fig. 9. $[\mathrm{Mn} / \mathrm{Fe}]$ vs. $[\mathrm{Fe} / \mathrm{H}]$ : present results, shown with the same symbols as in Fig. 7, compared with the literature: a) Feltzing et al. (2007) and b) Reddy et al. $(2003,2006)$ for thin disc (full green squares) and thick disc (red crosses) stars; c) Cayrel et al. (2004) (open red triangles) and Nissen \& Schuster (2011) (open red circles) for halo stars.

\subsection{Comparison with bulge Mn abundances from the literature}

In Fig. $7 \mathrm{~b}$ the present results are compared with literature Mn abundances for bulge giants, including bulge field stars by McWilliam et al. $(2003 \mathrm{a}, \mathrm{b})^{6}$, and three stars of NGC 6528, with stellar parameters derived by Zoccali et al. (2004), and Mn abundances derived by Sobeck et al. (2006) from the same spectra. The present Mn abundances appear to be in very good agreement with these data points both for field bulge giants (McWilliam et al. 2003a,b), and for the bulge globular cluster stars (Sobeck et al. 2006).

These data, together with the present results confirm a good agreement with the chemical evolution model by Cescutti et al. (2008). On the other hand the lack of a well-defined [Mn/Fe] trend in the range $-0.7 \lesssim[\mathrm{Fe} / \mathrm{H}] \lessgtr-0.2$, or even up to $[\mathrm{Fe} / \mathrm{H}] \sim 0.0$ if the McWilliam et al. field stars and NGC 6528 data (Sobeck et al.) are taken into account, does not allow the exclusion of the behaviour indicated in the models by TS98 and Timmes et al. (1995).

\subsection{Comparison with the Galactic thin disc, thick disc, and halo}

In Figs. 9 and 10 our results are plotted again, with different symbols identifying the four fields and the Baade's Window red

\footnotetext{
6 Mn abundances reported by McWilliam et al. (2003a,b) for the Sagittarius dwarf galaxy are not considered here, since this galaxy has a very different star formation history.
}

clump stars (as in Figs. 7a,b), now compared with literature data, for the thin disc, thick disc, and halo stars.

In Fig. 9a, a comparison with $\mathrm{Mn}$ abundances in thin and thick disc stars by Feltzing et al. (2007) indicates that the bulge giants show good agreement with both thin and thick disc stars for metallicities higher than $[\mathrm{Fe} / \mathrm{H}] \gtrsim-0.7$, whereas for lower abundances the $[\mathrm{Mn} / \mathrm{Fe}]$ values in the bulge appear to decrease faster than for the few thick disc stars with $[\mathrm{Fe} / \mathrm{H}]<-0.7$. The comparison with thin and thick disc stars by Reddy et al. (2003, 2006) given in Fig. 9b shows a decrease in $[\mathrm{Mn} / \mathrm{Fe}]$ for the thick disc stars that is more consistent with that observed for our bulge sample despite a rather large spread.

Figure $9 \mathrm{c}$ shows a mean $[\mathrm{Mn} / \mathrm{Fe}] \sim-0.4$ abundance in very metal-poor halo stars, from Cayrel et al. (2004), and $[\mathrm{Mn} / \mathrm{Fe}] \sim-0.3$, in moderately metal-poor halo stars from Nissen et al. (2011). A slope between the most metal-poor and the moderately metal-poor halo stars could be present. The two stars BW-f4 and BW-f8, at $[\mathrm{Fe} / \mathrm{H}] \sim-1.3$, have $[\mathrm{Mn} / \mathrm{Fe}] \sim-0.7$. However, they have $S / N=22$ and 38, respectively, in the original red spectra (Lecureur et al. 2007), therefore not as high as needed to claim a difference compared to other samples. In the star B3-b1 at $[\mathrm{Fe} / \mathrm{H}] \sim-0.8$, one line indicates a low Mn abundance, in contrast to the other three lines which show moderate Mn deficiencies; therefore, we gave a low weight to the discrepant line, only lowering the mean by -0.05 dex.

In Fig. 10 our results are compared with those by Ishigaki et al. (2013), Sobeck et al. (2006), Gratton (1989), and Prochaska et al. (2000), adopting for the last their mean values for standard model atmospheres. Ishigaki et al. (2013) derived 

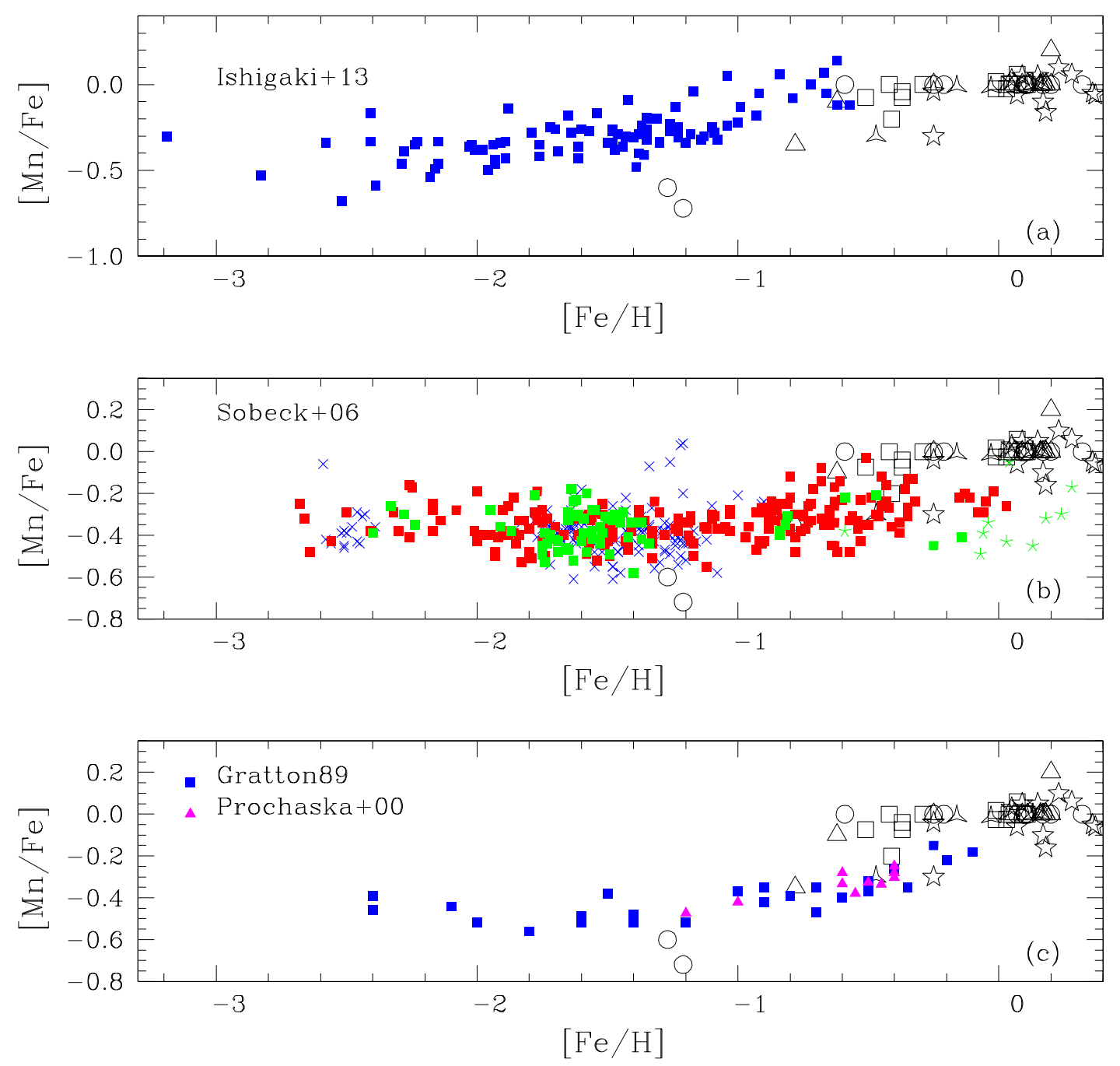

Fig. 10. $[\mathrm{Mn} / \mathrm{Fe}]$ vs. $[\mathrm{Fe} / \mathrm{H}]$ : present results, with the same symbols as in Fig. 7, compared with a) Ishigaki et al. (2013): thick disc and halo (blue squares); b) Sobeck et al. (2006): globular clusters (blue crosses); field stars (red squares), bulge metal-rich globular clusters plus the old open cluster Cr261 with stellar parameters from the literature (green stars); c) Gratton (1989) (blue squares), and Prochaska et al. (2000) (thick disc: full magenta triangles).

the abundances of iron-peak elements including $\mathrm{Mn}$ in 97 thick disc, inner and outer halo stars. Their $[\mathrm{Mn} / \mathrm{Fe}]$ ratios (Fig. 10a) start to drop from the solar value at $[\mathrm{Fe} / \mathrm{H}] \sim-0.7$, down to $[\mathrm{Mn} / \mathrm{Fe}] \sim-0.3$ in the range $-1.6 \lesssim[\mathrm{Fe} / \mathrm{H}] \lesssim-1.1$, and drop lower for the more metal-poor stars. The data could be interpreted as a steady increase from $[\mathrm{Mn} / \mathrm{Fe}] \sim-0.6$ at $[\mathrm{Fe} / \mathrm{H}] \sim$ -3.0 up to $[\mathrm{Mn} / \mathrm{Fe}] \sim 0.0$ at $[\mathrm{Fe} / \mathrm{H}] \sim-0.7$. The pace of decrease of $[\mathrm{Mn} / \mathrm{Fe}]$ runs in parallel with our bulge metal-poor stars, but with higher values. Sobeck et al. (2006) derived Mn abundances for a large number of field stars, and stars in globular clusters, and in one old open cluster. In particular for 200 stars in 19 globular clusters in the metallicity range $-2.7<[\mathrm{Fe} / \mathrm{H}]<$ -0.7 , an approximately constant value is found with a mean $[\mathrm{Mn} / \mathrm{Fe}]=-0.37$. Somewhat lower values of $[\mathrm{Mn} / \mathrm{Fe}] \sim-0.4$ were found for field stars by Gratton (1989) (Fig. 10c), Cayrel et al. (2004) (Fig. 9c), and Ishigaki et al. (2013) (Fig. 10a), and $[\mathrm{Mn} / \mathrm{Fe}] \approx-0.5$ by Reddy et al. (2006) (Fig. 9b). A few stars at metallicities $-1.6<[\mathrm{Fe} / \mathrm{H}]<-1.0$ show $[\mathrm{Mn} / \mathrm{Fe}] \sim-0.6$, compatible with the results for our bulge stars. Prochaska et al. (2000) analysed ten thick disc stars (Fig. 10c) in the metallicity range $-1.2 \lessgtr[\mathrm{Fe} / \mathrm{H}] \lessgtr-0.4$. Their $[\mathrm{Mn} / \mathrm{Fe}] \sim-0.3$ at $[\mathrm{Fe} / \mathrm{H}] \sim-0.4$ drops to $[\mathrm{Mn} / \mathrm{Fe}] \sim-0.4$ at $[\mathrm{Fe} / \mathrm{H}] \sim-1.2$, in good agreement with the present results for the metal-poor bulge giants.

Del Peloso et al. (2005) derived Mn abundances for 20 thin disc stars, with results compatible with those by Feltzing et al. (2007) and Reddy et al. (2003). Allen \& Porto de Mello (2011) derived $\mathrm{Mn}$ abundances in barium stars.

A further inspection of the data can be obtained by considering oxygen instead of iron as reference element, given that oxygen is a bona fide primary element produced uniquely by SNe II. The same applies to magnesium, however there is a possibility that some magnesium is produced in SNe Ia. As a matter of fact, Feltzing et al. (2007) were able to better disentangle thick disc from thin disc stars, through this approach. The oxygen abundances for the present sample of stars were derived in Zoccali et al. (2006) and Lecureur et al. (2007), with uncertainties of \pm 0.15 in $[\mathrm{O} / \mathrm{H}]$. These data are used to plot $[\mathrm{Mn} / \mathrm{O}]$ vs. $[\mathrm{Fe} / \mathrm{H}]$ and vs. $[\mathrm{O} / \mathrm{H}]$ (Figs. 11a,b). These figures disentangle the stellar populations more effectively, showing that the present bulge stars have a $[\mathrm{Mn} / \mathrm{O}]$ behaviour similar to that of the thick disc, extending the thick disc trend to higher metallicites (both in $[\mathrm{Fe} / \mathrm{H}]$ and $[\mathrm{O} / \mathrm{H}]$ ), and distinct from the thin disc trend at all metallicities. In terms of Mn production, Fig. $11 \mathrm{~b}$ has interesting 
B. Barbuy et al.: Manganese abundances in Galactic bulge red giants
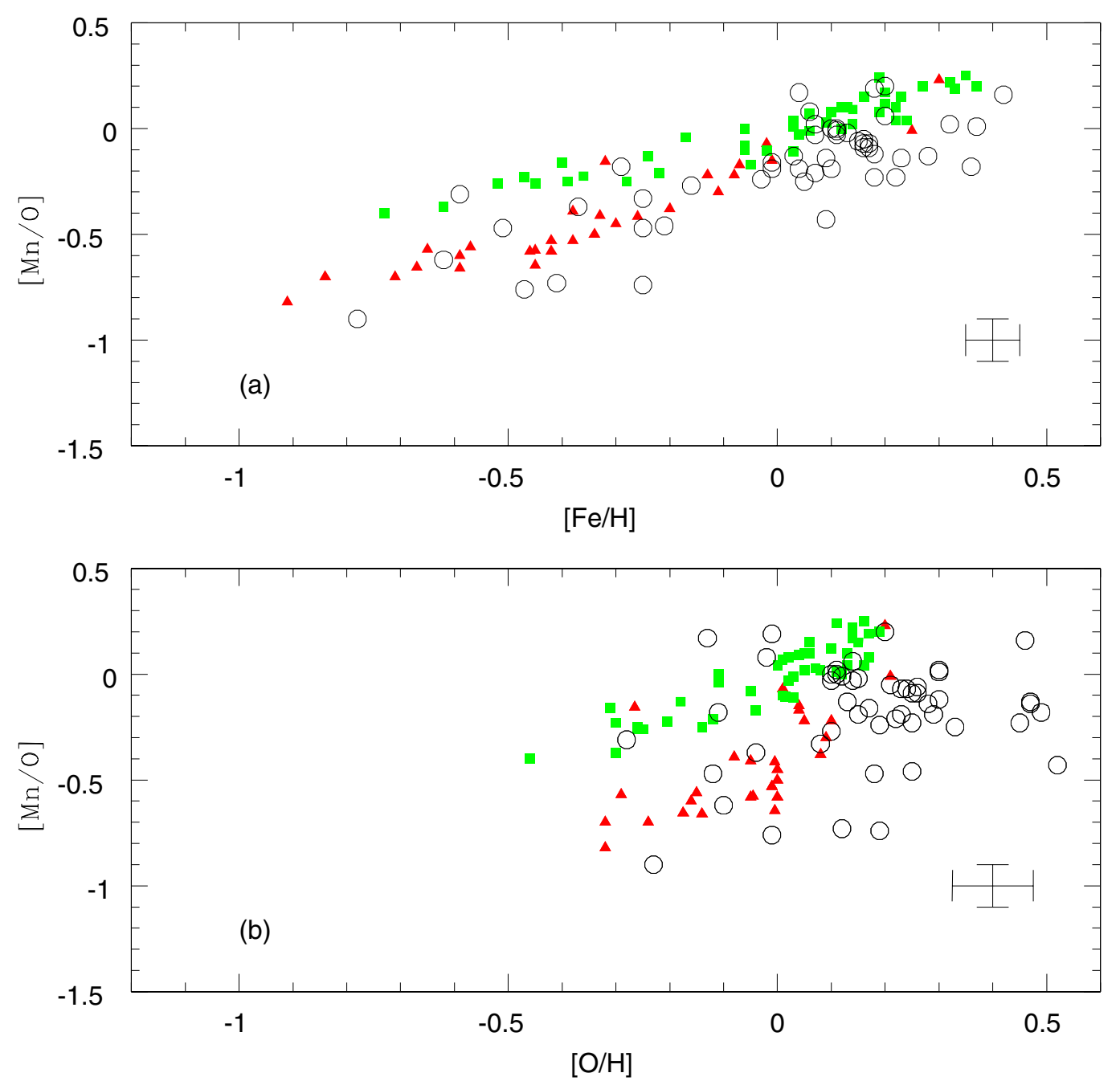

Fig. 11. a) $[\mathrm{Mn} / \mathrm{O}]$ vs. $[\mathrm{Fe} / \mathrm{H}]$; b) $[\mathrm{Mn} / \mathrm{O}]$ vs. $[\mathrm{O} / \mathrm{H}]$ : present results (open circles), compared with results from Feltzing et al. (2007) with same symbols as in Fig. 9a for thin disc (green squares) and thick disc (red full triangles) stars. Errorbars are given on the bottom-right corner of both panels.

implications since it should show more directly the metallicity dependence of Mn production, as well as the SNe Ia production of Mn. Cescutti et al. (2008) claim that both SNe II and $\mathrm{SNe}$ Ia metallicity dependent yields are needed to explain the Mn chemical evolution of the solar neighborhood, the Galactic bulge, and the Sgr dSph; if this were the case, one would typically expect two regimes of chemical enrichments: (i) dominated by $\mathrm{SN}$ II at low metallicities, possibly with a slope of $[\mathrm{Mn} / \mathrm{O}]$ with $[\mathrm{O} / \mathrm{H}]$ reflecting the metallicity dependence of the SNe II yields, followed by (ii) an enrichment dominated by $\mathrm{SNe} \mathrm{Ia}$, possibly with a slope reflecting the metallicity dependence of the SNe Ia yields. North et al. (2012) discuss these contributions further in the context of dwarf spheroidal galaxies (see their Fig. 7). The transition between the two regimes should occur at different $[\mathrm{O} / \mathrm{H}]$ in the different populations, mirroring the decrease in $[\alpha / \mathrm{Fe}]$ at the metallicity reached by the system when the delayed $\mathrm{SNe}$ Ia yields enter into play in the chemical evolution. The slope in regime (ii) can also depend on the population since the $\mathrm{SNe}$ Ia contributing at a given $[\mathrm{O} / \mathrm{H}]$ can be of different metallicities. Given that $\alpha$-elements evolution of $\alpha$-elements in thick disc and bulge stars is similar (e.g. Alves-Brito et al. 2010; González et al. 2011, Hill et al. 2011), it is hence expected that the bulge follows the thick disc in regime (ii) when $\mathrm{SNe}$ Ia are contributing, i.e. up to metallicities of $[\mathrm{O} / \mathrm{H}] \sim 0.0$. In this same metallicity range, the thin disc already includes $\mathrm{SNe}$ Ia products and $[\mathrm{Mn} / \mathrm{O}]$ is hence larger. Beyond solar $[\mathrm{O} / \mathrm{H}]$, if the thick disc and bulge differ, it could be partly attributed to the metallicity dependent yields of SNe Ia. We note that the mean $[\mathrm{Mn} / \mathrm{O}]$ in the bulge does not continue to rise for $[\mathrm{O} / \mathrm{H}]>0.0$, as one could expect if the $\mathrm{SNe}$ Ia yields were strongly metallicity dependent. According to Cescutti et al. (priv. comm.), the sensitivity of bulge models to the SNe Ia metallicity dependency is, however, negligible, at least when the SNe Ia progenitors are formed with roughly solar metallicities, and using yields from Maeder (1992) for oxygen, in which stellar rotation decreases the oxygen yields possibly too extremely. It would be very interesting to compare the $\mathrm{Mn} / \mathrm{O}$ in the bulge and thick disc to chemical evolution models with various hypotheses on the metallicity dependency of SNe Ia yields, and different models of oxygen yields, such as those of Cescutti et al. (2008).

In conclusion, in the metal-poor range $-1.3<[\mathrm{Fe} / \mathrm{H}]<-0.8$, the present sample includes two stars that are slightly more Mnpoor than shown in the literature, except for a closer agreement with data from Prochaska et al. (2000) and Reddy et al. (2006). The increase towards solar abundances starts to occur at around $-1.0 \lesssim[\mathrm{Fe} / \mathrm{H}] \lessgtr-0.7$, reaching the solar value at solar metallicity, but showing a spread of $-0.3<[\mathrm{Mn} / \mathrm{Fe}]<0.0$ in the range $-0.7<[\mathrm{Fe} / \mathrm{H}]<-0.2$. We note that a spread is also seen 
in some dwarf spheroidals (North et al. 2012), and could be explained if two channels of SNe Ia exist (Cescutti, priv. comm.). The behaviour of $[\mathrm{Mn} / \mathrm{O}]$ vs. $[\mathrm{O} / \mathrm{H}]$ (Fig. 11b) seems to indicate that the present sample of bulge stars have had a different chemical enrichment history to thick disc stars.

\section{Discussion}

A chemical evolution model by Tsujimoto \& Shigeyama (1998) predicted that $[\mathrm{Mn} / \mathrm{Fe}]$ would decrease with decreasing metallicity for $[\mathrm{Fe} / \mathrm{H}] \lesssim-3.0$, and would otherwise be a constant $[\mathrm{Mn} / \mathrm{Fe}] \sim-0.4$ in the range $-3.0 \lesssim[\mathrm{Fe} / \mathrm{H}] \lesssim-1.0$, and would then increase to the solar value reached at solar metallicity. The present and literature results agree with these predictions of low $[\mathrm{Mn} / \mathrm{Fe}]$ in the halo, possibly with a slope, that starts to increase due to the contribution of SNe Ia at $-1.0<[\mathrm{Fe} / \mathrm{H}] \sim-0.7$.

Timmes et al. (1995) presented calculations of Mn production as a function of metallicity through production in massive stars, with a metallicity dependent yield due to the lower neutron excess in the metal-poor stars, whereas SNe Ia contribute significantly to the Mn abundance at higher metallicities. Their models predicted a lower constant value in the halo of $[\mathrm{Mn} / \mathrm{Fe}] \sim-0.6$, and the increase in $[\mathrm{Mn} / \mathrm{Fe}]$ from $[\mathrm{Mn} / \mathrm{Fe}] \sim-0.6$ at $[\mathrm{Fe} / \mathrm{H}] \sim$ -1.0 to the solar ratio at solar metallicity, in good agreement with the present results for the bulge stars.

The Galactic bulge models by Cescutti et al. (2008), that include metallicity dependent yields for both SNe II (WW95) and $\mathrm{SNe} \mathrm{Ia}$, appear to most accurately reproduce the present results, confirming previous work by McWilliam et al. (2003a,b).

The present results confirm conclusions from previous work, namely, a low $[\mathrm{Mn} / \mathrm{Fe}]$ in the halo and metal-poor thick disc stars with $[\mathrm{Fe} / \mathrm{H}] \lesssim-0.7$ indicating that these stars were enriched in Mn by type II SNe. The contribution from SN Ia starts operating at $-1.0 \lesssim[\mathrm{Fe} / \mathrm{H}] \lessgtr-0.7$, possibly being different for the bulge, thick disc, and thin disc, given some differences shown in Figs. 9-11.

\section{Conclusions}

Abundances of iron-peak elements in different environments are key ingredients constraining nucleosynthesis yields in type II SNe and type Ia SNe, as well as in chemical evolution models (e.g. Iwamoto et al. 1999). The relative abundances of several iron-peak elements (species) relative to iron in the Galactic halo, disc, and bulge, as well as in globular clusters and dwarf galaxies can constrain models. The behaviour of $[\mathrm{Mn} / \mathrm{Fe}]$ vs. $[\mathrm{Fe} / \mathrm{H}]$ shows that the iron-peak element $\mathrm{Mn}$ has not been produced in the same conditions as even- $Z$ iron-peak elements such as Fe and Ni.

Abundances of $\mathrm{Mn}$ in metal-poor halo stars show $[\mathrm{Mn} / \mathrm{Fe}] \sim$ -0.4 in the metallicity range $-3.0 \lesssim[\mathrm{Fe} / \mathrm{H}] \lesssim-1.5$ (Cayrel et al. 2004; Ishigaki et al. 2013) confirming its production in core collapse $\mathrm{SNe}$, similarly to the $\alpha$ and heavy elements. As noted by Gratton $(1989)[\mathrm{Mn} / \mathrm{Fe}] \approx-0.4$ show a symmetric behaviour relative to the $[\alpha / \mathrm{Fe}] \approx+0.4$ in the halo. The metallicity-dependent yields from WW95 essentially reproduce this behaviour.

The triggering of type Ia $\mathrm{SNe}$ seems to occur at around $-1.0 \lesssim[\mathrm{Fe} / \mathrm{H}] \lesssim-0.7$ and predicted yields reproduce satisfactorily the steady increase of $[\mathrm{Mn} / \mathrm{Fe}]$ in thick disc stars, reaching the solar value at around $-0.7 \lesssim[\mathrm{Fe} / \mathrm{H}] \lesssim-0.0$, as shown in Figs. 9 and 10. We note that the data by Feltzing et al. (2007) show higher abundances of $[\mathrm{Mn} / \mathrm{Fe}] \sim-0.2$ in metal-poor thick disc stars, whereas data by Gratton (1989) and Prochaska et al. (2000) reach the solar $[\mathrm{Mn} / \mathrm{Fe}]$ ratio at metallicities of $[\mathrm{Fe} / \mathrm{H}] \sim-0.2$.
Cescutti et al. (2008) were able to reproduce the behaviour of $[\mathrm{Mn} / \mathrm{Fe}]$ vs. $[\mathrm{Fe} / \mathrm{H}]$ for the seven bulge stars by McWilliam et al. (2003a,b) by adopting a metallicity dependent yield for massive stars (WW95) and introducing a metallicity dependency in the yields by Iwamoto et al. (1999) proportional to $Z^{0.65}$. We find that their model is the most suitable to fit the present data. On the other hand, given a spread in Mn-over-Fe abundances in the metallicity range $-0.7 \lesssim[\mathrm{Fe} / \mathrm{H}] \lesssim-0.2$, the higher $\mathrm{Mn}$ abundances at $[\mathrm{Fe} / \mathrm{H}] \sim-0.7$ indicated in the TS98 model, or lower values as predicted by Timmes et al. (1995) cannot be excluded.

Using oxygen as reference element, the $[\mathrm{Mn} / \mathrm{O}]$ trend runs parallel to the thick disc stars, at the bulge higher metallicities. However, at the highest $[\mathrm{O} / \mathrm{H}]$, the $[\mathrm{Mn} / \mathrm{O}]$ in the bulge stars does not seem to increase anymore, possibly indicating that the SNe Ia metallicity dependency is weak, or even absent. We caution, however, that the measurement of $[\mathrm{Mn} / \mathrm{O}]$ suffers from large errors in these stars (mostly because of the $\mathrm{O}$ abundance uncertainties).

Finally, the low $\mathrm{Mn}$ abundance at $[\mathrm{Fe} / \mathrm{H}] \sim-1.0$ is in good agreement with results from damped Lyman- $\alpha$ systems (e.g. Pettini 1999; Fig. 24 in Prochaska et al. 2000).

Acknowledgements. We are grateful to G. Cescutti for useful comments on chemical enrichment. B.B. acknowledges partial financial support by CNPq and FAPESP. M.Z. and D.M. are supported by Fondecyt Regular 1110393, and 1130196, the BASAL Center for Astrophysics and Associated Technologies PFB-06, the FONDAP Center for Astrophysics 15010003, Proyecto Anillo ACT-86, and the Chilean Ministry for the Economy, Development, and Tourism Programa Iniciativa Científica Milenio through grant P07-021-F, awarded to The Milky Way Millennium Nucleus. M.T. acknowledges FAPESP postdoctoral fellowship No. 2012/05142-5.

\section{References}

Allen, D. M., \& Porto de Mello, G. F. 2011, A\&A, 525, A63

Alves-Brito, A., Meléndez, J., Asplund, M., Ramírez, I., \& Yong, D. 2010, A\&A, 513, A35

Asplund, M., Grevesse, N., Sauval, A. J., \& Scott, P. 2009, ARA\&A, 47, 481

Ballero, S. K., Matteucci, F., Origlia, L., \& Rich, R. M. 2007, A\&A, 467, 123

Barbuy, B., Perrin, M.-N., Katz, D., et al. 2003, A\&A, 404, 661

Bergemann, M., \& Gehren, T. 2008, A\&A, 492, 823

Biehl, D. 1976, Ph.D. Thesis, Kiel University

Blackwell-Whitehead, R., \& Bergemann, M. 2007, A\&A, 472, L43

Booth, A. J., Blackwell, D. E., Petford, A. D., \& Shallis, M. J. 1984, MNRAS, 208, 147

Bravo, E. 2013, A\&A, 550, A24

Brodzinski, T., Kronfeldt, H.-D., Kropp, J.-R., \& Winkler, R. Z. 1987, Z. Phys. D, 7, 161

Cayrel, R., Depagne, E., Spite, M., et al. 2004, A\&A, 416, 1117

Cescutti, G., Matteucci, F., Lanfranchi, G. A., \& McWilliam, A. 2008, A\&A, 491, 401

del Peloso, E. F., Cunha, K., da Silva, L., \& Porto de Mello, G. F. 2005, A\&A, 441, 1149

Den Hartog, E. A., Lawler, J. E., \& Sobeck, J. S. 2011, ApJS, 194, 35

Feltzing, S., Fohlman, M., \& Bensby, T. 2007, A\&A, 467, 665

Gratton, R. G. 1989, A\&A, 208, 171

Grevesse, N., \& Sauval, J. N. 1998, Space Sci. Rev., 35, 161

González, O. A., Rejkuba, M., Zoccali, M., et al. 2011, A\&A, 530, A54

Gustafsson, B., Edvardsson, B., Eriksson, K., et al. 2008, A\&A, 486, 951

Handrich, E., Steudel, A., \& Walther, H. 1969, Phys. Lett. A, 29, 486

Hill, V., Lecureur, A., Gómez, A., et al. 2011, A\&A, 534, A80

Hinkle, K., Wallace, L., Valenti, J., \& Harmer, D. 2000, Visible and Near Infrared Atlas of the Arcturus Spectrum 3727-9300 A (San Francisco: ASP)

Ishigaki, M. N., Aoki, W., \& Chiba, M. 2013, ApJ, 771, 67

Iwamoto, K., Brachwitz, F., Nomoto, K., et al. 1999, ApJS, 125, 439

Kobayashi, C., Umeda, H., Nomoto, K., Tominaga, N., \& Ohkubo, T. 2006, ApJ, 643, 1145

Kurúcz, R. 1993, CD-ROM 23

Lecureur, A., Hill, V., Zoccali, M., et al. 2007, A\&A, 465, 799

Lodders, K., Palme, H., \& Gail, H.-P. 2009, Landolt-Börnstein - Group VI Astronomy and Astrophysics Numerical Data and Functional Relationships in Science and Technology Volume 4B: Solar System, ed. J. E. Trümper, 4.4., 44 
B. Barbuy et al.: Manganese abundances in Galactic bulge red giants

Maeder, A. 1992, A\&A, 264, 105

Martin, W. C., Fuhr, J. R., Kelleher, D. E., et al. 2002, NIST Atomic Database (version 2.0), http://physics.nist.gov/asd, National Institute of Standards and Technology, Gaithersburg, MD

Matteucci, F., \& Greggio, L. 1986, A\&A, 154, 279

McWilliam, A., Rich, R. M., \& Smecker-Hane, T. A. 2003a, ApJ, 592, L21

McWilliam, A., Rich, R. M., \& Smecker-Hane, T. A. 2003b, ApJ, 593, L145

Meléndez, J., Barbuy, B., Bica, E., et al. 2003, A\&A, 411, 417

Modigliani, A., Mulas, G., Porceddu, I., et al. 2004, The Messenger, 118, 8

Nakamura, T., Umeda, H., Nomoto, K., Thielemann, F.-K., \& Burrows, A. 1999, ApJ, 517, 193

Nissen, P. E., \& Schuster, W. J. 2011, A\&A, 530, A15

Nissen, P. E., Chen, Y. Q., Schuster, W. J., \& Zhao, G. 2000, A\&A, 353, 722

Nomoto, K., Thielemann, F.-K., \& Yokoi, Y. 1984, ApJ, 286, 644

Nomoto, K., Hashimoto, M., Tsujimoto, T., et al. 1997, Nucl. Phys. A, 616, 79

North, P., Cescutti, G., Jablonka, P., et al. 2012, A\&A, 541, A45

Pettini, M., Ellison, S. L., Steidel, C. C., \& Bowen, D. V. 1999, ApJ, 510, 576

Piskunov, N., Kupka, F., Ryabchikova, T., Weiss, W., \& Jeffery, C. 1995, A\&AS, 112,525

Prochaska, J. X., \& McWilliam, A. 2000, ApJ, 537, L57
Prochaska, J. S., Naumov, S. O., Carney, B. W., McWilliam, A., \& Wolfe, A. M. 2000, AJ, 120, 2513

Ramírez, S., \& Allende Prieto, C. 2011, ApJ, 743, 135

Reddy, B. E., Tomkin, J., Lambert, D. L., \& Allende Prieto, C. 2003, MNRAS, 340,304

Reddy, B. E., Lambert, D. L., \& Allende Prieto, C. 2006, MNRAS, 367, 1329

Ryde, N., Gustafsson, B., Edvardsson, E., et al. 2010, A\&A, 509, A20

Sobeck, J. S., Ivans, I. I., Simmerer, J. A., et al. 2006, AJ, 131, 2949

Thielemann, F.-K., Nomoto, K., \& Yokoi, Y. 1986, A\&A, 158, 17

Thielemann, F.-K., Nomoto, K., \& Hashimoto, M. 1996, ApJ, 460, 408

Timmes, F. X., Woosley, S. E., \& Weaver, T. A. 1995, ApJS, 98, 617

Tsujimoto, T., \& Shigeyama, T. 1998, ApJ, 508, L151 (TS98)

Umeda, H., \& Nomoto, K. 2002, ApJ, 565, 385 (UN02)

Umeda, H., \& Nomoto, K. 2005, ApJ, 619, 427

Woodgate, G. K., \& Martin, J. S. 1957, Proc. Phys. Soc. Lon. A, 70, 485

Woosley, S. E., \& Weaver, T. A. 1995, ApJS, 101, 181 (WW95)

Yoshii, Y., Tsujimoto, T., \& Kawara, K. 1998, ApJ, 507, L113

Zoccali, M., Barbuy, B., Hill, V., et al. 2004, A\&A, 423, 507

Zoccali, M., Lecureur, A., Barbuy, B., et al. 2006, A\&A, 457, L1

Zoccali, M., Lecureur, A., Hill, V., et al. 2008, A\&A, 486, 177

Pages 10 to 14 are available in the electronic edition of the journal at http://www. aanda.org 
Table 1. Atomic constants used to compute hyperfine structure from Handrich et al. (1969), Biehl (1976), and Brodzinski et al. (1987).

\begin{tabular}{lrrrrrrrrr}
\hline \hline Species & $\lambda(\AA)$ & Lower level & $J$ & $\mathrm{~A}(\mathrm{MHz})$ & $\mathrm{B}(\mathrm{MHz})$ & Upper level & $J$ & $\mathrm{~A}(\mathrm{MHz})$ & $\mathrm{B}(\mathrm{MHz})$ \\
\hline \multirow{2}{*}{ MnI } & 6013.513 & $3 \mathrm{~d}^{5} 4 \mathrm{~s} 4 \mathrm{p}$ & 1.5 & 571.85 & 11.5 & $3 \mathrm{~d}^{5} 4 \mathrm{~s} 5 \mathrm{~s}$ & 2.5 & 808.54 & 0. \\
& 6016.640 & $3 \mathrm{~d}^{5} 4 \mathrm{~s} 4 \mathrm{p}$ & 2.5 & 466.94 & -72.93 & $3 \mathrm{~d}^{5} 4 \mathrm{~s} 5 \mathrm{~s}$ & 2.5 & 808.54 & 0. \\
& 6021.800 & $3 \mathrm{~d}^{5} 4 \mathrm{~s} 4 \mathrm{p}$ & 3.5 & 429.165 & 66.6 & $3 \mathrm{~d}^{5} 4 \mathrm{~s} 5 \mathrm{~s}$ & 2.5 & 808.54 & 0. \\
& 5394.669 & $3 \mathrm{~d}^{5} 4 \mathrm{~s}^{2}$ & 2.5 & -71.950 & 0. & $3 \mathrm{~d}^{5} 4 \mathrm{~s} 4 \mathrm{p}$ & 3.5 & 545.622 & 101.930 \\
\hline
\end{tabular}

Notes. $B=0$ for $3 \mathrm{~d}^{5} 4 \mathrm{~s}^{2}$ was adopted when not given in the literature.

Table 2. Central wavelengths and total literature and adopted oscillator strengths.

\begin{tabular}{lrrrrrrrrr}
\hline \hline Species & $\lambda(\AA)$ & $\chi_{\text {ex }}(\mathrm{eV})$ & \multicolumn{1}{c}{$g f_{\text {Booth }}$} & $g f_{\text {Kurucz }}$ & $g f_{\text {NIST }}$ & $g f_{\text {VALD }}$ & $g f_{\text {B-HB07 }}$ & $g f_{\text {DenHartog }}$ & $g f_{\text {adopted }}$ \\
\hline MnI & 6013.513 & 3.072451 & -0.251 & -0.251 & -0.252 & -0.252 & -0.43 & -0.352 & -0.40 \\
& 6016.640 & 3.073534 & - & -0.216 & - & 0.0 & -0.183 & -0.25 & -0.216 \\
& 6021.800 & 3.075294 & 0.034 & 0.034 & 0.035 & 0.035 & -0.054 & -0.12 & -0.10 \\
& 5394.67 & 0.0 & -3.503 & -3.591 & -3.503 & -3.503 & - & - & -3.55 \\
\hline
\end{tabular}

Table 3. Hyperfine structure for Mn I lines.

\begin{tabular}{cccccccc}
\hline \hline $\begin{array}{c}6013.488 \AA ; \\
\log g f(\text { total })=-0.40\end{array}$ & \multicolumn{2}{c}{$\begin{array}{c}6016.673 \AA ; \\
\log g f(\text { total })=-0.266\end{array}$} & \multicolumn{2}{c}{$\begin{array}{c}6021.792 \AA ; \\
\log g f(\text { total })=-0.10\end{array}$} & $\begin{array}{c}5394.669 \AA ; \chi=0.0 \mathrm{eV} \\
\log g f(\text { total })=-3.55\end{array}$ \\
\hline$\lambda(\AA)$ & $\log \mathrm{gf}$ & $\lambda(\AA)$ & $\log g f$ & $\lambda(\AA)$ & $\log g f$ & $\lambda(\AA)$ & $\log g f$ \\
\hline 6013.559 & -1.9563 & 6016.665 & -1.8223 & 6021.829 & -1.6563 & 5394.722 & -5.1063 \\
6013.549 & -1.6341 & 6016.681 & -1.8223 & 6021.819 & -1.6263 & 5394.722 & -5.0763 \\
6013.529 & -1.8102 & 6016.671 & -2.5882 & 6021.799 & -2.4045 & 5394.712 & -4.8211 \\
6013.563 & -2.0021 & 6016.651 & -1.5602 & 6021.828 & -1.3711 & 5394.724 & -5.8545 \\
6013.543 & -1.4792 & 6016.683 & -1.5602 & 6021.809 & -1.4502 & 5394.714 & -4.9002 \\
6013.514 & -1.4000 & 6016.664 & -1.8892 & 6021.779 & -2.3253 & 5394.699 & -4.5992 \\
6013.564 & -2.1782 & 6016.634 & -1.4579 & 6021.823 & -1.1492 & 5394.716 & -5.7753 \\
6013.535 & -1.4580 & 6016.681 & -1.4579 & 6021.794 & -1.3833 & 5394.701 & -4.8333 \\
6013.496 & -1.1270 & 6016.652 & -1.4421 & 6021.755 & -2.4502 & 5394.681 & -4.4152 \\
6013.563 & -2.5584 & 6016.613 & -1.4633 & 6021.814 & -0.9652 & 5394.704 & -5.9002 \\
6013.524 & -1.6041 & 6016.675 & -1.4633 & 6021.775 & -1.4089 & 5394.684 & -4.8589 \\
6013.475 & -0.9149 & 6016.636 & -1.1111 & 6021.726 & -2.8024 & 5394.657 & -4.2568 \\
& & 6016.587 & -1.6260 & 6021.801 & -0.8068 & 5394.687 & -6.2524 \\
& & 6016.663 & -1.6260 & 6021.752 & -1.5849 & 5394.661 & -5.0349 \\
\hline
\end{tabular}


B. Barbuy et al.: Manganese abundances in Galactic bulge red giants

Table 4. Atmospheric parameters adopted from Zoccali et al. (2006) and Lecureur et al. (2007) for the red giants, and from Hill et al. (2011) for the red clump stars, and abundances of $[\mathrm{Mn} / \mathrm{Fe}]$ derived in the present work.

\begin{tabular}{|c|c|c|c|c|c|c|c|c|c|c|c|c|}
\hline Star & OGLE ${ }^{\circ}$ & $\begin{array}{r}\alpha(\mathrm{J} 2000) \\
(\mathrm{h}, \mathrm{m}, \mathrm{s})\end{array}$ & $\begin{array}{r}V \\
{[\mathrm{mag}]}\end{array}$ & $\begin{array}{l}T_{\text {eff }} \\
{[\mathrm{K}]}\end{array}$ & $\log g$ & {$[\mathrm{Fe} / \mathrm{H}]$} & $\begin{array}{c}v_{\mathrm{t}} \\
{\left[\mathrm{kms}^{-1}\right]}\end{array}$ & {$[\mathrm{Mn}$} & & & & $\begin{array}{r}\mathrm{Mn} / \mathrm{Fe}] \\
\text { mean }\end{array}$ \\
\hline B6-b1 & $29280 \mathrm{c} 3$ & $180950.604-314051.599$ & 16.14 & 4400 & 1.8 & 0.07 & 1.6 & 0.20 & 0.00 & 0.00 & - & 0.06 \\
\hline B6-b2 & $83500 \mathrm{c} 6$ & $181034.117-314909.094$ & 16.40 & 4200 & 1.5 & -0.01 & 1.4 & - & -0.05 & 0.00 & - & -0.03 \\
\hline B6-b3 & $31220 \mathrm{c} 2$ & $181019.183-314028.102$ & 16.09 & 4700 & 2.0 & 0.10 & 1.6 & 0.00 & 0.00 & 0.00 & - & 0.00 \\
\hline B6-b4 & $60208 \mathrm{c} 7$ & $181007.901-315241.288$ & 16.12 & 4400 & 1.9 & -0.41 & 1.7 & 0.00 & -0.25 & -0.25 & -0.30 & -0.20 \\
\hline B6-b5 & $31090 \mathrm{c} 2$ & $181037.509-314029.098$ & 16.04 & 4600 & 1.9 & -0.37 & 1.3 & 0.00 & -0.05 & 0.00 & -0.10 & -0.04 \\
\hline B6-b6 & $77743 \mathrm{c} 7$ & $180949.217-315007.597$ & 16.09 & 4600 & 1.9 & 0.11 & 1.8 & 0.00 & 0.00 & 0.00 & - & 0.00 \\
\hline B6-b8 & $108051 \mathrm{c} 7$ & $180956.070-314546.301$ & 16.29 & 4100 & 1.6 & 0.03 & 1.3 & - & -0.05 & 0.00 & - & -0.03 \\
\hline B6-f1 & $23017 \mathrm{c} 3$ & $181004.591-314145.295$ & 15.96 & 4200 & 1.6 & -0.01 & 1.5 & 0.05 & 0.00 & 0.00 & - & 0.02 \\
\hline B6-f2 & $90337 \mathrm{c} 7$ & $181011.636-314819.196$ & 15.91 & 4700 & 1.7 & -0.51 & 1.5 & 0.00 & 0.00 & 0.00 & -0.30 & -0.08 \\
\hline B6-f3 & $21259 \mathrm{c} 2$ & $181017.850-314155.196$ & 15.71 & 4800 & 1.9 & -0.29 & 1.3 & 0.00 & 0.00 & 0.00 & 0.00 & 0.00 \\
\hline B6-f5 & $33058 \mathrm{c} 2$ & $181041.643-314011.801$ & 15.90 & 4500 & 1.8 & -0.37 & 1.4 & 0.00 & 0.00 & 0.00 & -0.30 & -0.08 \\
\hline B6-f7 & $100047 \mathrm{c} 6$ & $181052.430-314642.097$ & 15.95 & 4300 & 1.7 & -0.42 & 1.6 & - & 0.00 & 0.00 & - & 0.00 \\
\hline B6-f8 & $1653 \mathrm{c} 3$ & $963-314322.503$ & 15.65 & 4900 & 1.8 & 0.04 & 1.6 & 0.00 & 0.00 & 0.00 & - & 0.00 \\
\hline BW-b2 & 214192 & 300557.797 & 6.58 & 4300 & 1.9 & 0.22 & 1.5 & 0.00 & 0.00 & 0.00 & - & 0.00 \\
\hline BW-b4 & 545277 & $180405.476-300552.496$ & 6.95 & 4300 & 1.4 & 0.07 & 1.4 & 0.00 & 0.00 & 0.00 & - & 0.00 \\
\hline BW-b5 & 82760 & $180413.407-295817.800$ & 6.64 & 4000 & 1.6 & 0.17 & 1.2 & 0.00 & 0.00 & 0.0 & - & 0.0 \\
\hline BW-b6 & 392931 & $.969-300627.900$ & 6.42 & 4200 & 1.7 & -0.25 & 1.3 & 0.00 & 0.00 & 0.00 & -0.50 : & 0.00 \\
\hline BW-b7 & 554694 & $180404.693-300239.604$ & 16.69 & 4200 & 1.4 & 0.10 & 1.2 & 0.00 & - & 0.00 & - & 0.00 \\
\hline BW-f1 & 433669 & $180337.268-295422.294$ & 16.14 & 4400 & 1.8 & 0.32 & 1.6 & 0.00 & 0.00 & 0.00 & - & 0.00 \\
\hline BW-f4 & 537070 & $180401.528-301020.700$ & 16.07 & 4800 & 1.9 & -1.21 & 1.7 & -0.30 & -0.80 & -0.60 & -0.90 & -0.72 \\
\hline BW-f5 & 240260 & $180439.753-295519.794$ & 15.88 & 4800 & 1.9 & -0.59 & 1.3 & 0.00 & 0.00 & 0.00 & -0.50 & 0.00 \\
\hline BW-f6 & 392918 & $180337.014-300704.299$ & 16.37 & 4100 & 1.7 & -0.21 & 1.5 & 0.00 & 0.00 & 0.00 & -0.50 : & 0.00 \\
\hline BW-f7 & 357480 & $80444.045-300315.193$ & 16.31 & 4400 & 1.9 & 0.11 & 1.7 & - & 0.00 & 0.00 & - & 0.00 \\
\hline BW-f8 & 44598 & $0330.615-300144.803$ & 16.00 & 5000 & 2.2 & -1.27 & 1.8 & -0.60 & -0.60 & -0.60 & -0.60 & -0.60 \\
\hline BL-1 & $58 \mathrm{c} 3$ & $8.643-343315.241$ & 15.37 & 4500 & 2.1 & -0.16 & 1.5 & 0.00 & -0.03 & 0.00 & - & -0.01 \\
\hline BL-3 & $1859 \mathrm{c} 2$ & $10-343159.353$ & 5.53 & 4500 & 2.3 & -0.03 & 1.4 & 0.0 & -0.05 & 0.00 & - & -0.02 \\
\hline BL-4 & $3328 \mathrm{c} 6$ & $1.240-344448.217$ & 4.98 & 4700 & 2.0 & 0.13 & 1.5 & 0.00 & 0.00 & 0.00 & - & 0.00 \\
\hline BL-5 & $1932 \mathrm{c} 2$ & $18-343147.913$ & 5.39 & 4500 & 2.1 & 0.16 & 1.6 & 0.00 & 0.00 & 0.00 & - & 0.00 \\
\hline BL-7 & $336 \mathrm{c} 7$ & $92-3438$ & 5.33 & 4700 & 2.4 & -0.47 & 1.4 & -0.30 & -0.30 & -0.30 & -0.30 & -0.30 \\
\hline B3-b1 & $132160 \mathrm{C} 4$ & $180815.971-254209.801$ & 6.35 & 4300 & 1.7 & -0.78 & 1.5 & -0.30 & -0.30 & -0.90 & -0.30 & -0.35 \\
\hline B3-b2 & $262018 C 7$ & $.192-255647.300$ & 6.63 & 4500 & 2.0 & 0.18 & 1.5 & - & 0.00 & 0.00 & - & 0.00 \\
\hline B3-b3 & $90065 \mathrm{C} 3$ & $180846.527-254244.401$ & 16.59 & 4400 & 2.0 & 0.18 & 1.5 & - & 0.00 & 0.00 & - & 0.00 \\
\hline B3-b4 & $215681 \mathrm{C} 6$ & $180844.597-255756.802$ & 16.36 & 4500 & 2.1 & 0.17 & 1.7 & 0.00 & 0.00 & 0.00 & - & 0.00 \\
\hline B3-b5 & $286252 C 7$ & $180900.644-254806.699$ & 16.23 & 4600 & 2.0 & 0.11 & 1.5 & 0.00 & 0.00 & 0.00 & - & 0.00 \\
\hline B3-b7 & $282804 C 7$ & $180916.670-254926.006$ & 16.36 & 4400 & 1.9 & 0.20 & 1.3 & 0.00 & 0.00 & 0.00 & - & 0.00 \\
\hline B3-b8 & 240083C6 & $180824.733-254844.300$ & 16.49 & 4400 & 1.8 & -0.62 & 1.4 & -0.10 & -0.30 & 0.00 & 0.00 & -0.10 \\
\hline B3-f1 & 129499C4 & $180816.301-254319.104$ & 16.32 & 4500 & 1.9 & 0.04 & 1.6 & 0.00 & 0.00 & 0.00 & - & 0.00 \\
\hline B3-f2 & $259922 \mathrm{C} 7$ & $180915.730-255732.701$ & 16.54 & 4600 & 1.9 & -0.25 & 1.8 & 0.00 & 0.00 & 0.00 & - & 0.00 \\
\hline B3-f3 & $424 \mathrm{C} 3$ & $0849.747-254036.898$ & 16.32 & 4400 & 1.9 & 0.06 & 1.7 & 0.00 & 0.00 & 0.00 & - & 0.00 \\
\hline B3-f4 & 208959C6 & $180844.419-260025.001$ & 16.51 & 4400 & 2.1 & 0.09 & 1.5 & 0.00 & - & 0.00 & - & 0.00 \\
\hline B3-f5 & 49289C2 & $180918.531-254337.403$ & 6.61 & 4200 & 2.0 & 0.16 & 1.8 & 0.00 & 0.00 & 0.00 & - & 0.00 \\
\hline B3-f7 & 279577C7 & $180923.818-255038.104$ & 6.28 & 4800 & 2.1 & 0.16 & 1.7 & 0.00 & 0.00 & 0.00 & & 0.00 \\
\hline & $193190 \mathrm{C} 5$ & $7-255004.4$ & 6.26 & 4800 & 1.9 & 0.20 & 1. & 0.30 & 0.00 & 0.30 & - & 0.20 \\
\hline Wc-1 & & $5-30053$ & 5.84 & 4476 & 2.1 & 0.09 & 1.5 & 0.00 & 0.00 & 0.15 & - & 0.05 \\
\hline Wc-2 & 545749 & $4-300537.390$ & 7.19 & 4558 & 2.2 & 0.18 & 1.2 & 0.10 & -0.30 & -0.30 & - & -0.16 \\
\hline $\mathrm{c}-3$ & 4840 & $0-3001$ & 6.91 & 4513 & 2.1 & 0.28 & 1. & 0.00 & 0.00 & 0.00 & - & 0.06 \\
\hline Wc-4 & 564857 & $180355.416-300057.314$ & 6.76 & 4866 & 2.2 & 0.05 & 1.3 & 0.10 & 0.00 & 0.00 & - & 0.03 \\
\hline Wc-5 & 575542 & $180356.021-295543.716$ & 16.98 & 4535 & 2.1 & 0.42 & 1.5 & 0.30 & 0.00 & 0.30 & - & 0.20 \\
\hline Wc-6 & 575585 & $180356.543-295511.787$ & 16.74 & 4769 & 2.2 & -0.25 & 1.3 & -0.10 & 0.00 & -0.05 & -0.02 & -0.04 \\
\hline BWc-7 & 577 & $180356.543-295511.787$ & 17.01 & 4590 & 2.2 & -0.25 & 1.1 & -0.30 & - & -0.30 & - & -0.30 \\
\hline BWc-8 & 78255 & $180312.494-300359.111$ & 16.97 & 4610 & 2.2 & 0.37 & 1.3 & 0.10 & -0.30 & 0.00 & - & -0.06 \\
\hline BWc-9 & 78271 & $180316.683-300351.406$ & 16.90 & 4539 & 2.1 & 0.15 & 1.5 & 0.20 & -0.05 & 0.00 & - & 0.05 \\
\hline BWc-10 & 89589 & $180318.914-300109.983$ & 16.70 & 4793 & 2.2 & 0.07 & 1.3 & -0.10 & -0.10 & 0.00 & - & -0.06 \\
\hline BWc-11 & 89735 & $180304.749-295935.301$ & 16.69 & 4576 & 2.1 & 0.17 & 1.0 & -0.10 & -0.10 & -0.10 & - & -0.10 \\
\hline BWc-12 & 89832 & $180320.102-295825.785$ & 16.92 & 4547 & 2.1 & 0.23 & 1.3 & 0.30 & 0.00 & 0.00 & - & 0.10 \\
\hline BWc-13 & 89848 & $180304.612-295814.080$ & 16.73 & 4584 & 2.1 & 0.36 & 1.1 & 0.30 & -0.30 & -0.15 & - & -0.05 \\
\hline
\end{tabular}

Notes. Mn1/Mn2/Mn3/Mn4 correspond to the 6013/6016/6021/5394 ̊̊ lines. 
A\&A 559, A5 (2013)

Table 5. Uncertainties on derived $[\mathrm{Mn} / \mathrm{Fe}]$ for the metal-poor star B3-b1 and the red clump star BWc-6.

\begin{tabular}{lrrrrrrrrrr}
\hline \hline Star & $T_{\text {eff }}$ & $\log g$ & {$[\mathrm{Fe} / \mathrm{H}]$} & $v_{\mathrm{t}}$ & Ref. & {$[\mathrm{Mn} 1 / \mathrm{Fe}]$} & {$[\mathrm{Mn} 2 / \mathrm{Fe}]$} & {$[\mathrm{Mn} 3 / \mathrm{Fe}]$} & {$[\mathrm{Mn} 4 / \mathrm{Fe}]$} & {$[\mathrm{Mn} / \mathrm{Fe}]$} \\
\hline B3-b1 & 4300 & 1.7 & -0.78 & 1.5 & 1 & -0.3 & -0.3 & $(-0.9)$ & -0.3 & -0.35 \\
B3-b1 & 4400 & 1.7 & -0.60 & 1.3 & 2 & -0.3 & -0.3 & $(-1.0)$ & -0.40 & -0.38 \\
B3-b1 & 4365 & 2.0 & -0.73 & 1.5 & 3 & -0.3 & -0.3 & $(-0.9)$ & -0.35 & -0.37 \\
B3-b1 & 4150 & 1.7 & -0.78 & 1.5 & - & -0.4 & -0.45 & $(-1.0)$ & -0.5 & -0.50 \\
B3-b1 & 4300 & 2.2 & -0.78 & 1.5 & - & -0.3 & -0.3 & $(-0.8)$ & -0.3 & -0.35 \\
B3-b1 & 4300 & 1.7 & -0.78 & 1.6 & - & -0.3 & -0.3 & $(-0.9)$ & -0.6 & -0.35 \\
B3-b1 & 4300 & 1.7 & -0.88 & 1.5 & - & -0.15 & -0.15 & $(-0.7)$ & -0.15 & -0.20 \\
\hline BWc-6 & 4787 & 2.2 & -0.35 & 1.5 & 1 & 0.0 & +0.1 & 0.0 & 0.0 & 0.03 \\
BWc-6 & 4769 & 2.2 & -0.17 & 1.2 & 4 & -0.05 & -0.05 & -0.1 & -0.1 & -0.08 \\
BWc-6 & 4769 & 2.2 & -0.25 & 1.3 & 5 & -0.1 & 0.0 & -0.05 & -0.02 & -0.04 \\
BWc-6 & 4620 & 2.2 & -0.25 & 1.3 & - & 0.0 & -0.3 & -0.2 & -0.25 & -0.19 \\
BWc-6 & 4769 & 2.7 & -0.25 & 1.3 & - & 0.0 & 0.0 & 0.0 & 0.0 & 0.00 \\
BWc-6 & 4769 & 2.2 & -0.25 & 1.4 & - & 0.0 & 0.0 & 0.0 & 0.0 & 0.00 \\
BWc-6 & 4769 & 2.2 & -0.35 & 1.3 & - & 0.0 & 0.0 & 0.0 & 0.0 & 0.00 \\
\hline
\end{tabular}

References. (1) Lecureur et al. (2007) (present results); (2) Zoccali et al. (2008); (3) Ryde et al. (2010); (4) Hill et al. (2011) GIRAFFE data; (5) Hill et al. (2011) UVES data (present results). [Mn/Fe] is also derived with changes in model parameters of $\Delta T_{\mathrm{eff}}=-150 \mathrm{~K}, \Delta \log g=0.2$, $\Delta v_{\mathrm{t}}=0.1 \mathrm{~km} \mathrm{~s}^{-1}, \Delta[\mathrm{Fe} / \mathrm{H}]=-0.1$ dex. We note that star B3-b1 was chosen for its reanalysis by Ryde et al. (2010), but it has one discardable line that gives a low Mn (in parenthesis) to which a low weight is given since it disagrees with the other three lines, and only an extra -0.05 dex is added to the mean of the other three lines.

Table 6. Uncertainties on the derived $[\mathrm{Mn} / \mathrm{Fe}]$ value for model changes of $\Delta T_{\text {eff }}=-150 \mathrm{~K}, \Delta \log g=0.2, \Delta v_{\mathrm{t}}=0.1 \mathrm{~km} \mathrm{~s}{ }^{-1}, \Delta[\mathrm{Fe} / \mathrm{H}]=-0.1 \mathrm{dex}$, and corresponding total error.

\begin{tabular}{lrrrrrr}
\hline \hline Star & {$[\mathrm{Fe} / \mathrm{H}]$} & $\Delta T$ & $\Delta \log g$ & $\Delta[\mathrm{Fe} / \mathrm{H}]$ & $\Delta v_{\mathrm{t}}$ & $\left(\sum \mathrm{x}^{2}\right)^{1 / 2}$ \\
& & $(-150 \mathrm{~K})$ & $(+0.2 \mathrm{dex})$ & $(-0.1 \mathrm{dex})$ & $\left(+0.1 \mathrm{~km} \mathrm{~s}^{-1}\right)$ & \\
$(1)$ & $(2)$ & $(3)$ & $(4)$ & $(5)$ & $(6)$ & \\
\hline B3-b1 & -0.78 & -0.09 & +0.01 & +0.10 & -0.03 & 0.14 \\
BWc-6 & -0.25 & -0.17 & +0.01 & +0.02 & +0.02 & 0.17 \\
\hline
\end{tabular}


B. Barbuy et al.: Manganese abundances in Galactic bulge red giants
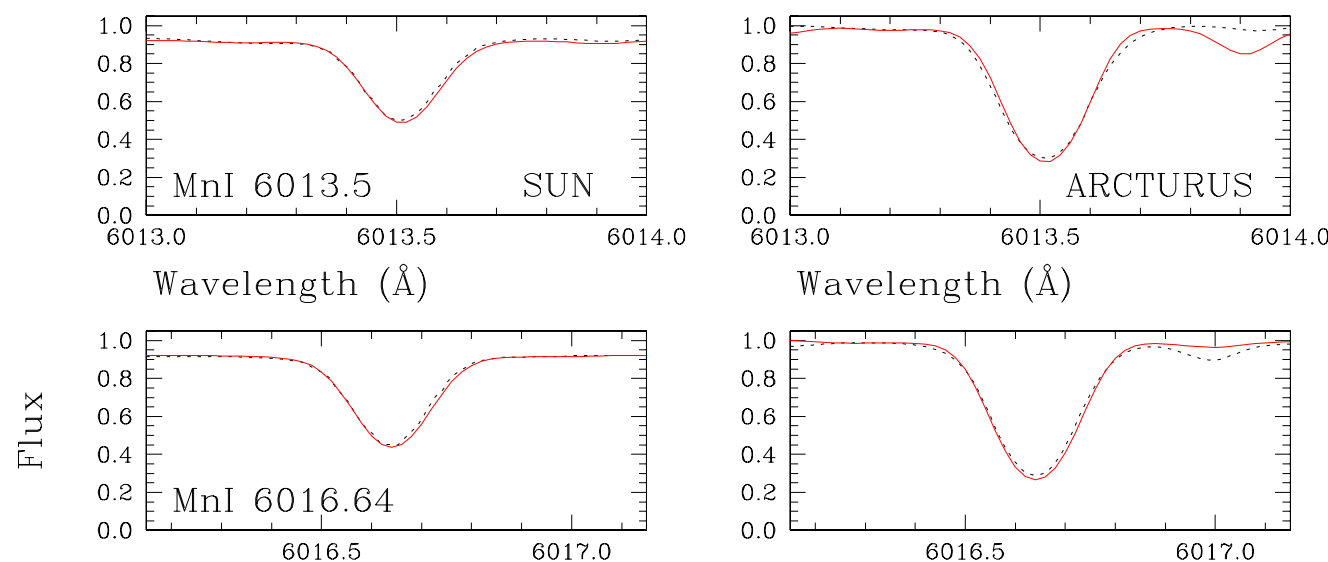

Wavelength $(\AA)$

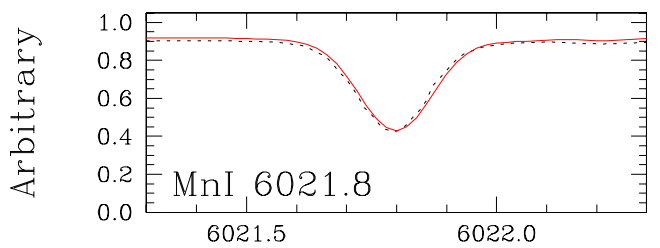

Wavelength $(\AA)$

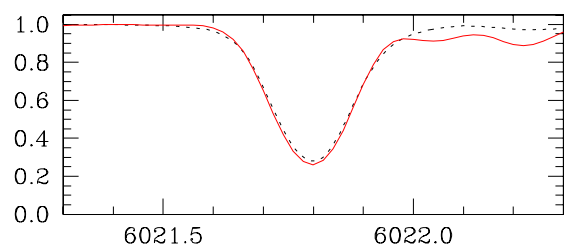

Wavelength $(\AA)$

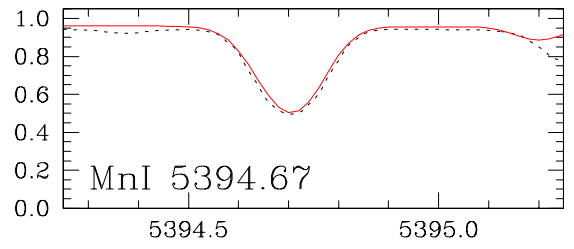

Wavelength $(\AA)$

Wavelength $(\AA)$

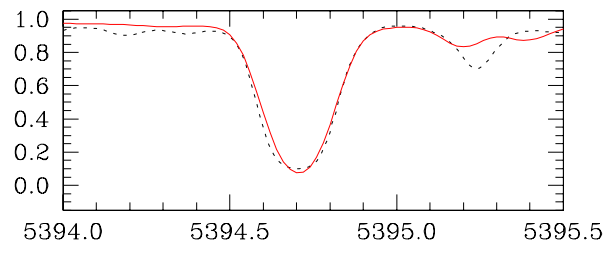

Wavelength $(\AA)$

Fig. 1. Lines of Mn I fittings on the solar spectrum observed with the UVES spectrograph, and on the Arcturus spectrum (Hinkle et al. 2000). Observed spectrum (dashed lines); synthetic spectra (solid red line).

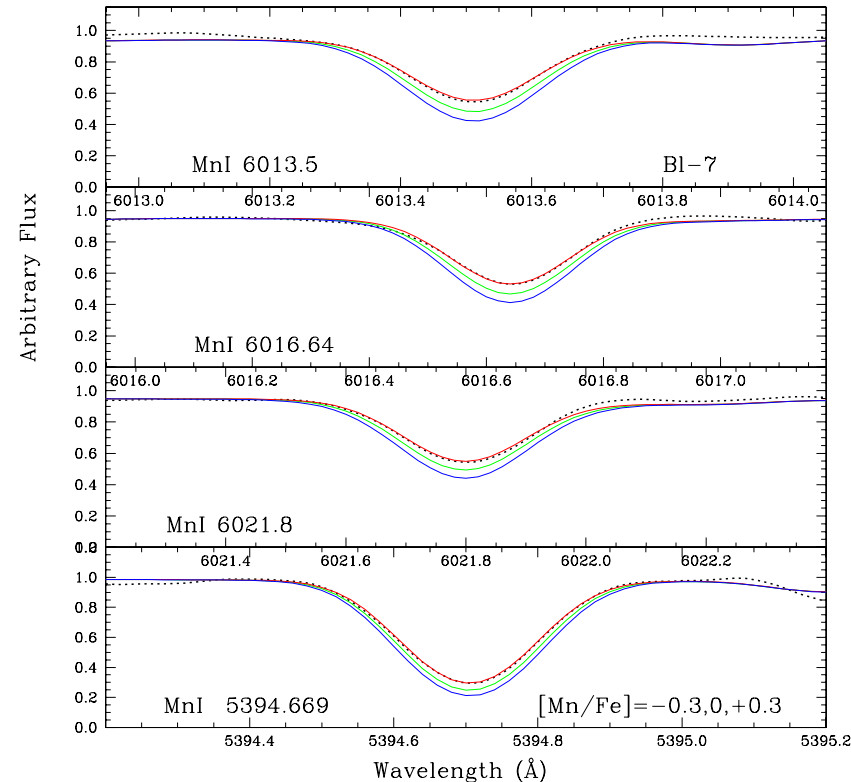

Fig. 2. Fits of the four Mn I lines for the metal-poor $([\mathrm{Fe} / \mathrm{H}]=-0.47)$ star BL-7.

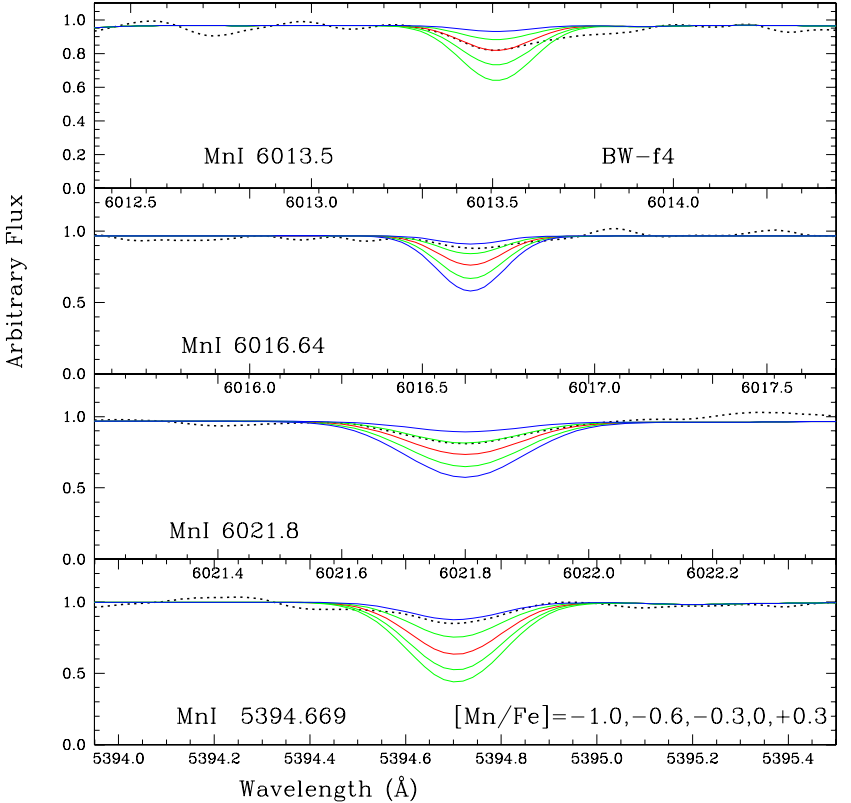

Fig. 3. Fits of the four Mn I lines for the metal-poor $([\mathrm{Fe} / \mathrm{H}]=-1.21)$ star BW-f4. 
A\&A 559, A5 (2013)

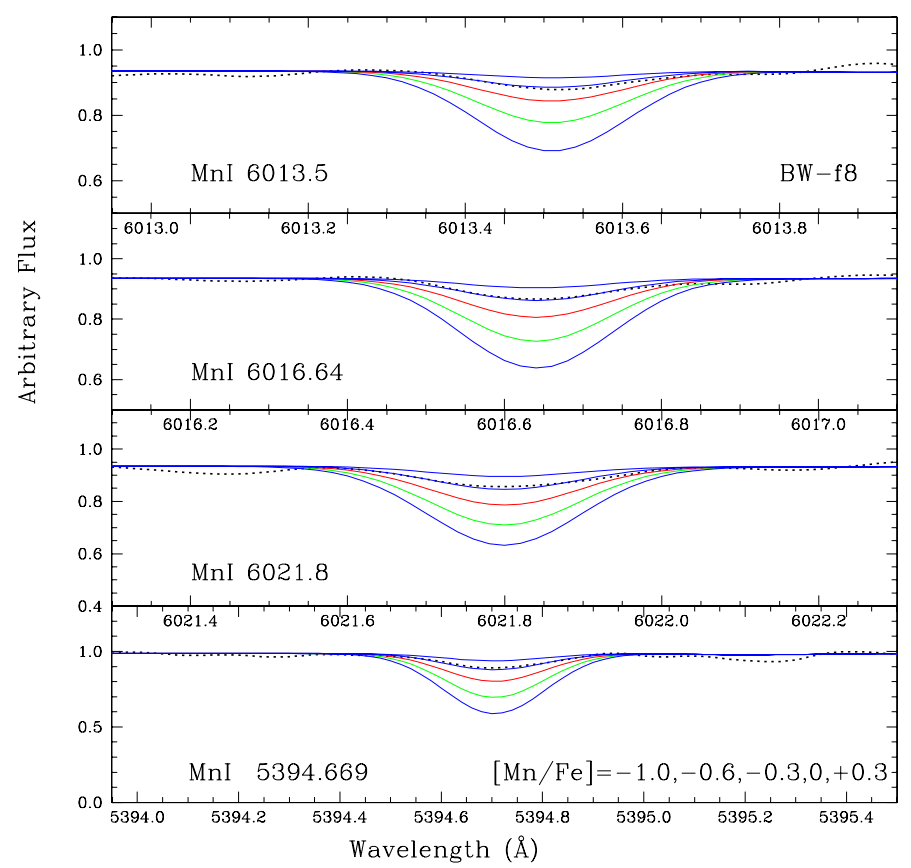

Fig. 4. Fits of the four MnI lines for the most metal-poor $([\mathrm{Fe} / \mathrm{H}]=$ -1.27) sample star BW-f8.

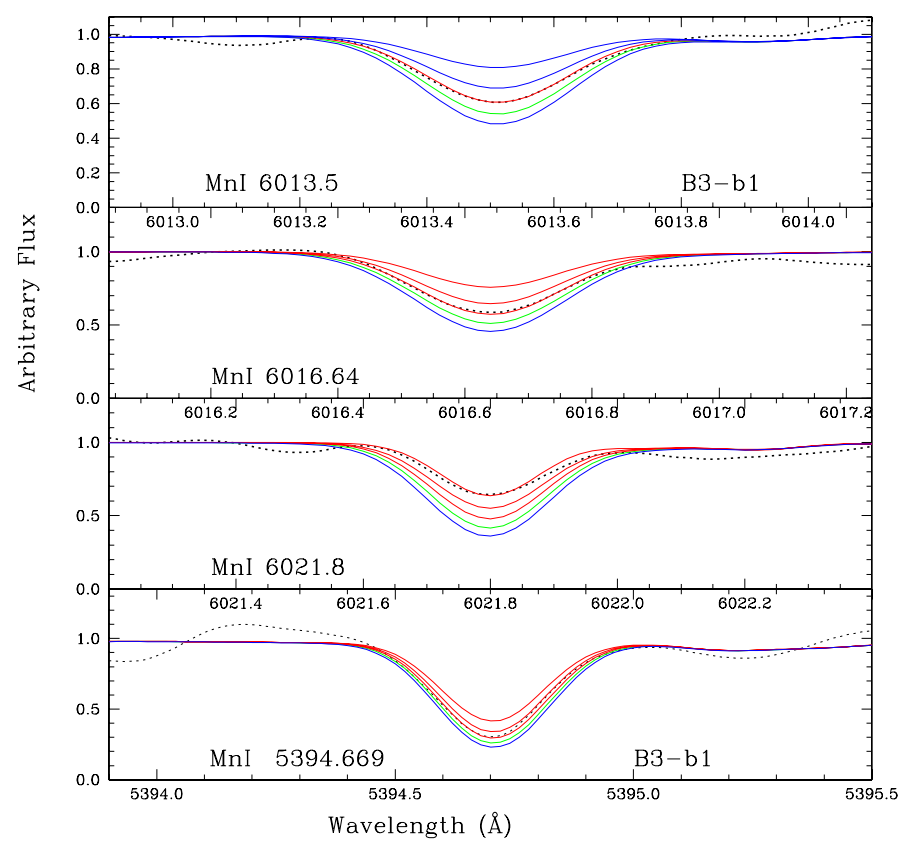

Fig. 5. Fits of the four MnI lines for the metal-poor $([\mathrm{Fe} / \mathrm{H}]=-0.78)$ star B3-b1.

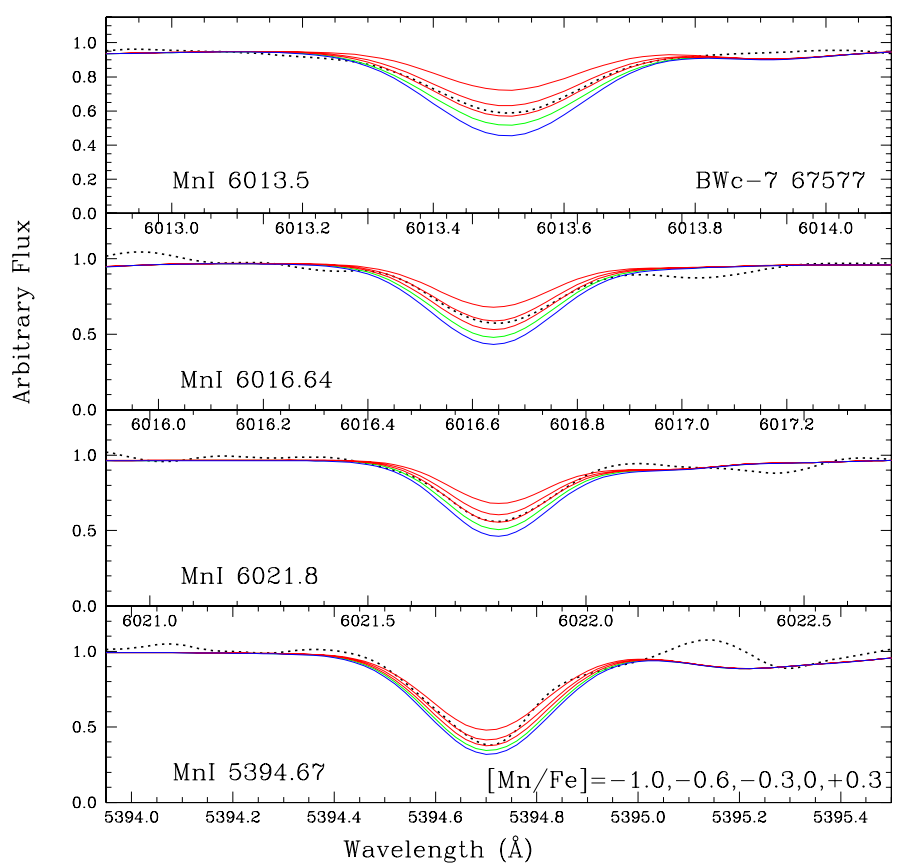

Fig. 6. Fits of the four $\mathrm{MnI}$ lines for the $([\mathrm{Fe} / \mathrm{H}]=-0.25)$ red clump star BWc-7. 